ESAIM: M2AN 55 (2021) S29-S64

https://doi.org/10.1051/m2an/2020021
ESAIM: Mathematical Modelling and Numerical Analysis

www.esaim-m2an.org

\title{
ON A CLASS OF SPLINES FREE OF GIBBS PHENOMENON
}

\author{
Sergio Amat ${ }^{1}$, Juan Ruiz ${ }^{1, *}$, Chi-Wang Shu² and Juan Carlos Trillo ${ }^{1}$
}

\begin{abstract}
When interpolating data with certain regularity, spline functions are useful. They are defined as piecewise polynomials that satisfy certain regularity conditions at the joints. In the literature about splines it is possible to find several references that study the apparition of Gibbs phenomenon close to jump discontinuities in the results obtained by spline interpolation. This work is devoted to the construction and analysis of a new nonlinear technique that allows to improve the accuracy of splines near jump discontinuities eliminating the Gibbs phenomenon. The adaption is easily attained through a nonlinear modification of the right hand side of the system of equations of the spline, that contains divided differences. The modification is based on the use of a new limiter specifically designed to attain adaption close to jumps in the function. The new limiter can be seen as a nonlinear weighted mean that has better adaption properties than the linear weighted mean. We will prove that the nonlinear modification introduced in the spline keeps the maximum theoretical accuracy in all the domain except at the intervals that contain a jump discontinuity, where Gibbs oscillations are eliminated. Diffusion is introduced, but this is fine if the discontinuity appears due to a discretization of a high gradient with not enough accuracy. The new technique is introduced for cubic splines, but the theory presented allows to generalize the results very easily to splines of any order. The experiments presented satisfy the theoretical aspects analyzed in the paper.
\end{abstract}

Mathematics Subject Classification. 65D05, 65D17, 65M06, 65N06.

Received April 8, 2019. Accepted March 28, 2020.

\section{INTRODUCTION}

A spline can be defined as any function constructed from one or more polynomial pieces that are joined together satisfying given differentiability requirements. These functions are widely used in the industry for computational aided geometric design where splines are used to represent geometric entities. Some applications of splines can be found in $[1,13,18,20,21,23,31]$ and the references therein. When working with splines without multiple knots, it happens that in the presence of discontinuities in the function or its derivatives, numerical artifacts always come into scene. Also, in industrial applications where the modelization is done through the use of discretization grids, numerical discontinuities appear close to high gradients when the grid is not fine enough. In these cases it is usually needed an $a d$ hoc local refinement of the grid in order to avoid numerical artifacts.

Keywords and phrases. Splines, adaption to discontinuities, interpolation, computer aided design (modeling of curves).

1 Department of Applied Mathematics and Statistics, Universidad Politécnica de Cartagena, Cartagena, Spain.

2 Division of Applied Mathematics, Brown University, Providence, RI 02912, USA.

*Corresponding author: juan.ruiz@upct.es 
Splines have also been widely used for function approximation, where the same kind of problems appear when dealing with discontinuous functions.

In the literature about interpolation and approximation, the classical approach consists in using linear methods, see for example Chapter 1 of [21] or [31] and the references therein. Automatic adaption to data with low regularity is offered by nonlinear approximation and wavelet methods, see [22]. Other approaches, following Harten's path of constructing nonlinear reconstruction operators, Amat et al. $[6,8,9,11,12]$ introduce a nonlinear processing of data that attains adaption to discontinuities, although none of this papers deals directly with the theoretical elimination of Gibbs effects. In [26,27], the author proposes nonlinear multiresolution methods for data compression and interpolation. In [28,29], the authors apply nonlinear shock capturing schemes to solve PDEs that arise from conservation laws. In $[2,4,8,15-17]$, the authors adapt these methods to function approximation and image processing using multiresolution schemes. It seems that, when working with splines, the more accepted approach is to directly construct a piecewise polynomial function of the chosen order using the available data, not taking into account the possible discontinuities that can be contained in these data. This strategy directly leads to the apparition of Gibbs effect $[24,30,39]$ in the reconstructed function. The solution that we propose to solve some of these problems is to introduce a nonlinear treatment of the right hand side of the system of equations that determines the spline. The modification is designed to attain adaption automatically when discontinuities in the function occur. Other approaches to eliminate Gibbs phenomenon, although without automatic adaption to discontinuities, have been addressed before in previous articles [32,37].

The problem that arises from using linear techniques is related to the accuracy of the approximation near the discontinuities: the order of approximation is lost due to Gibbs effect and diffusion. Any stencil that touches the discontinuity will be affected and the approximation will be inaccurate. Because of this fact, the increasing of the length of the stencil will not improve the approximation accuracy and will result in larger regions affected around discontinuities. A solution for this problem is to choose stencils that do not cross discontinuities. This is the key idea beneath ENO (Essentially Non Oscillatory) interpolation, first introduced by Harten et al. [28, 29] in the context of conservation laws. Using ENO strategy, the regions affected by discontinuities are reduced to an interval that contains the discontinuity. This is possible because ENO strategy selects among several, the stencil that is not affected by the discontinuity. The interested reader can refer to $[4,5,16,19,28,29,33-36]$ and the references therein in order to obtain more information about ENO. Unfortuntatelly, this strategy is not designed to work with splines. In [14], the authors try to adapt splines detecting the position of discontinuities and breaking the spline in several pieces. In this article we will use a different strategy: to introduce a nonlinear modification in the spline such that adaption is attained automatically without using any explicit detection of discontinuities. The motivation of this strategy is that it is imposible to know the exact position of jump discontinuities from the sampling of a function, as the exact position of this kind of discontinuities is lost during the process of discretization. Thus, it is convenient to design strategies to attain adaption to discontinuities even when the exact position of the discontinuity can not be known. As mentioned before, in [14] the authors propose a strategy to adapt splines to the presence of corner singularities in the point values using a detection technique. The mentioned strategy can not be used for jump discontinuities in the point values, as it is not possible to localize the position of the discontinuity. It can not be used neither with numerical discontinuities, i.e. when the sampling rate is not high enough to capture high gradients. In these cases the resultant discontinuity is considered numeric, as it is produced by the discretization process, and the reconstruction should recover the diffusion that was originally present in the continuous function, but avoiding Gibbs oscillations. If the function is contaminated with noise, the technique presented in [14] losses its effectiveness. This is due to the fact that noise reduces the original regularity of the data, if that data has been obtained from the sampling of a function with certain regularity. The technique in [14] is precisely based on the regularity of the data, as it uses differences or divided differences to detect the presence of discontinuities. If noise is present, the new technique presented in this article can be an option to attain adaption to discontinuities.

Summarizing, in this article it is our aim to design a new class of nonlinear splines adapted to the presence of discontinuities in the function, such that Gibbs effect is eliminated in the reconstructed function close to this kind of discontinuities. We will see that the problem is not trivial and that, in order to solve it, many 
considerations must be taken into account. The objective is to attain adaption close to jump discontinuities while maintaining the order of accuracy reached by cubic splines at smooth zones. The core of the new method will be based on the design of a nonlinear weighted mean with suitable adaption properties close to jump discontinuities. As far as we know, it is the first time that this kind of mean appears in the literature. It is based on a generalization for non uniform grids of the already existing limiters introduced in [33] and called $p$-power means. The new method is robust even in the presence of noise and the results obtained numerically confirm the theoretical aspects analyzed in the paper.

The present work is organized as follows: Section 2 describes the discretization that we will use, explains how classical splines are obtained and how to adapt them to the presence of discontinuities. Section 3 introduces and analyzes the properties of a new nonlinear weighted mean that is used to construct a new class of splines adapted to the presence of jump discontinuities. Section 4 studies the approximation properties of the new class of splines close to discontinuities. Section 5 presents some numerical experiments where it is shown how the new nonlinear splines perform close to jump discontinuities with uniform and non uniform grid spacing. Section 6 presents some conclusions. Finally, Appendix A analyzes the accuracy of cubic splines close to discontinuities and Appendix B gives a bound for the overshoot of cubic splines close to the jump of a step function.

\section{Classical CUBic splines}

In the literature, many references can be found about splines, for example [21] treats the field from a practical point of view and presents an extensive bibliography for the interested reader. In this work, we will try to build a sequence of polynomials of degree three in order to obtain a piecewise polynomial function that is $C^{2}$ : a cubic spline. Functions with $C^{2}$ regularity are the most used in CAD (computational aided design) and industrial design, as they present suitable properties for aerodynamics and hydrodynamics.

In this section we will introduce the classical cubic splines in order to obtain a self contained paper. From this construction we put some emphasis in the parts that allow to obtain a nonlinear spline adapted to discontinuities.

First of all, let us describe the discretization of the data that we will use along the article. Let's consider the set of piecewise continuous functions in the interval $[a, b]$, the space of finite sequences $V$ of length $N=m+1$ and let $X$ be a uniform or non-uniform partition of the interval $[a, b]$ in $m$ subintervals,

$$
X=\left\{x_{i}\right\}_{i=0}^{m}, \quad x_{0}=a, \quad h_{i}=x_{i}-x_{i-1} .
$$

Let's consider now the point-values discretization, that we define as,

$$
y_{i}=y\left(x_{i}\right), i=0, \ldots, m \text {. }
$$

In order to construct the spline $g(x)$ in the interval $[a, b]$, we will start from $m+1$ pairs of values $\left(x_{i}, y_{i}\right), i=$ $0, \ldots, m$ and the expression of the polynomial $g_{i}(x)$ at a particular interval $\left[x_{i}, x_{i+1}\right]$, such that $g(x)=g_{i}(x), x \in$ $\left[x_{i}, x_{i+1}\right]$,

$$
g_{i}(x)=a_{i}\left(x-x_{i}\right)^{3}+b_{i}\left(x-x_{i}\right)^{2}+c_{i}\left(x-x_{i}\right)+d_{i} .
$$

According to spline theory the polynomial $g_{i}(x)$ satisfying

$$
\begin{aligned}
g_{i-1}\left(x_{i}\right) & =y_{i}, \\
g_{i-1}^{\prime}\left(x_{i}\right) & =g_{i}^{\prime}\left(x_{i}\right), \\
g_{i}\left(x_{i}\right) & =y_{i}, \\
g_{i-1}^{\prime \prime}\left(x_{i}\right) & =g_{i}^{\prime \prime}\left(x_{i}\right) .
\end{aligned}
$$

and

$$
\begin{aligned}
g_{0}\left(x_{0}\right) & =y_{0}, \\
g_{m-1}\left(x_{m}\right) & =y_{m},
\end{aligned}
$$


gives a cubic spline that is at least $C^{2}$. Solving the previous equations, we obtain that,

$$
\begin{aligned}
d_{i} & =y_{i}, \\
c_{i} & =D_{i}, \\
a_{i} & =\frac{h_{i+1} D_{i+1}+D_{i} h_{i+1}+2 y_{i}-2 y_{i+1}}{h_{i+1}^{3}}, \\
b_{i} & =-\frac{h_{i+1} D_{i+1}+2 D_{i} h_{i+1}+3 y_{i}-3 y_{i+1}}{h_{i+1}^{2}},
\end{aligned}
$$

where $D_{i}$ stand for the first derivatives at every interior knot.

In fact it is possible to express the polynomial spline in (2.1) in terms of the Hermite basis $\left\{b_{1}, b_{2}, b_{3}, b_{4}\right\}$ such that

$$
\begin{aligned}
& b_{1}\left(x_{i}\right)=1, b_{1}\left(x_{i+1}\right)=0, \quad b_{1}^{\prime}\left(x_{i}\right)=0, \quad b_{1}^{\prime}\left(x_{i+1}\right)=0, \\
& b_{2}\left(x_{i}\right)=0, b_{2}\left(x_{i+1}\right)=1, b_{2}^{\prime}\left(x_{i}\right)=0, b_{2}^{\prime}\left(x_{i+1}\right)=0, \\
& b_{3}\left(x_{i}\right)=0, \quad b_{3}\left(x_{i+1}\right)=0, b_{3}^{\prime}\left(x_{i}\right)=1, b_{3}^{\prime}\left(x_{i+1}\right)=0, \\
& b_{4}\left(x_{i}\right)=0, b_{4}\left(x_{i+1}\right)=0, b_{4}^{\prime}\left(x_{i}\right)=0, b_{4}^{\prime}\left(x_{i+1}\right)=1 .
\end{aligned}
$$

For the uniform case we get,

$$
\begin{aligned}
g_{i}(x) & =\left(h D_{i+1}+D_{i} h+2 y_{i}-2 y_{i+1}\right) s^{3}-\left(h D_{i+1}+2 D_{i} h+3 y_{i}-3 y_{i+1}\right) s^{2}+D_{i} h s+y_{i}, \\
& =\left(1-s^{2}(3-2 s)\right) y_{i}+s^{2}(3-2 s) y_{i+1}+\left(s^{3}-2 s^{2}+s\right) h D_{i}+\left(s^{3}-s^{2}\right) h D_{i+1} \\
& =b_{1}(s) y_{i}+b_{2}(s) y_{i+1}+b_{3}(s) h D_{i}+b_{4}(s) h D_{i+1}
\end{aligned}
$$

for $s=\frac{x-x_{i}}{h} \in[0,1]$.

Now, in order to compute the $D_{i}$ values, we have $m-1$ conditions due to the continuity requirement in the second derivative. Since we have to determine $m+1$ unknowns, two more conditions must be chosen for the boundary conditions. Several options are available in the literature. Some examples are: the natural cubic spline if we suppose that the second derivatives are equal to zero at the boundaries, the not-a-knot condition, that imposes the condition that the first and the last interior nodes at $x_{1}$ and $x_{n-1}$ are not knots anymore, the complete cubic spline if we impose slope conditions at the boundaries... In this work we have chosen the natural cubic spline, as we are not interested in the accuracy of the spline at the boundaries, but at points close to the discontinuity. Any other boundary condition might have been chosen. However, it is important to have in mind that the boundary conditions may affect the precision of the splines in the $l_{\infty}$ norm. The only boundary condition that assure maximum accuracy in infinity norm are those that imply using real data, i.e. for example true derivatives or second derivatives at the boundaries. Other conditions may imply order reduction close to the boundaries and, necessarily, order reduction in the infinity norm.

Let's obtain a system of equations for the $D_{i}$. Replacing the expression of the spline (2.1) in (2.2), we obtain,

$$
\frac{D_{i-1}}{h_{i}}+2\left(\frac{1}{h_{i}}+\frac{1}{h_{i+1}}\right) D_{i}+\frac{D_{i+1}}{h_{i+1}}=3\left(\frac{y_{i+1}-y_{i}}{h_{i+1}^{2}}+\frac{y_{i}-y_{i-1}}{h_{i}^{2}}\right) .
$$

Choosing now the natural boundary conditions, the second derivative at the beginning and the end of the spline must be zero. From (2.1) and assuming that $g_{0}^{\prime \prime}(0)=0$, this condition transforms into,

$$
2 D_{0}+D_{1}=3\left(\frac{y_{1}-y_{0}}{h_{1}}\right) .
$$

The expression for the right boundary can be obtained from $g_{m-1}^{\prime \prime}(1)=0$, that leads to the equation,

$$
D_{m-1}+2 D_{m}=3\left(\frac{y_{m}-y_{m-1}}{h_{m}}\right) .
$$


Thus, we will obtain the system,

$$
\begin{aligned}
& {\left[\begin{array}{lllllll}
2 & 1 & 0 & 0 & \cdots & 0 & 0 \\
\frac{1}{h_{1}} & 2\left(\frac{1}{h_{1}}+\frac{1}{h_{2}}\right) & \frac{1}{h_{2}} & 0 & \cdots & 0 & 0 \\
0 & \frac{1}{h_{2}} & 2\left(\frac{1}{h_{2}}+\frac{1}{h_{3}}\right) & \frac{1}{h_{3}} & \cdots & 0 & 0 \\
\cdots & \cdots & \cdots & \cdots & \cdots & \cdots & \cdots \\
0 & 0 & 0 & 0 & \cdots & 2\left(\frac{1}{h_{m-1}}+\frac{1}{h_{m}}\right) & \frac{1}{h_{m}} \\
0 & 0 & 0 & 0 & \cdots & 1
\end{array}\right]\left[\begin{array}{l}
D_{0} \\
D_{1} \\
D_{2} \\
\cdots \\
D_{m-1} \\
D_{m}
\end{array}\right]} \\
& =3 \cdot\left[\begin{array}{l}
\delta_{1} \\
\frac{\delta_{2}}{h_{2}}+\frac{\delta_{1}}{h_{1}} \\
\frac{\delta_{3}}{h_{3}}+\frac{\delta_{2}}{h_{2}} \\
\cdots \\
\frac{\delta_{m}}{h_{m}}+\frac{\delta_{m-1}}{h_{m-1}} \\
\delta_{m}
\end{array}\right],
\end{aligned}
$$

being $\delta_{i}=\frac{y_{i}-y_{i-1}}{h_{i}}$. The previous expression can be also written as,

$$
\begin{aligned}
& {\left[\begin{array}{lllllll}
2 & 1 & 0 & 0 & \cdots & 0 & 0 \\
\frac{1}{h_{1}} & 2\left(\frac{1}{h_{1}}+\frac{1}{h_{2}}\right) & \frac{1}{h_{2}} & 0 & \cdots & 0 & 0 \\
0 & \frac{1}{h_{2}} & 2\left(\frac{1}{h_{2}}+\frac{1}{h_{3}}\right) & \frac{1}{h_{3}} & \cdots & 0 & 0 \\
\cdots & \cdots & \cdots & \cdots & \cdots & \cdots & \cdots \\
0 & 0 & 0 & 0 & \cdots & 2\left(\frac{1}{h_{m-1}}+\frac{1}{h_{m}}\right) & \frac{1}{h_{m}} \\
0 & 0 & 0 & 0 & \cdots & 1 & 2
\end{array}\right]\left[\begin{array}{l}
D_{0} \\
D_{1} \\
D_{2} \\
\cdots \\
D_{m-1} \\
D_{m}
\end{array}\right]} \\
& =3 \cdot\left[\begin{array}{ll}
\delta_{1} & \\
\left(\frac{1}{h_{1}}+\frac{1}{h_{2}}\right) & \frac{\frac{\delta_{2}}{h_{2}}+\frac{\delta_{1}}{h_{1}}}{h_{1}+\frac{1}{h_{2}}} \\
\left(\frac{1}{h_{2}}+\frac{1}{h_{3}}\right) & \frac{\frac{\delta_{2}}{h_{2}}+\frac{\delta_{3}}{h_{3}}}{\frac{1}{h_{2}}+\frac{1}{h_{3}}} \\
\cdots & \frac{\delta_{m-1}}{h_{m-1}+\frac{\delta_{m}}{h_{m}}} \\
\left(\frac{1}{h_{m-1}}+\frac{1}{h_{m}}\right) & \frac{1}{h_{m-1}}+\frac{1}{h_{m}} \\
\delta_{m}
\end{array}\right],
\end{aligned}
$$

where the right hand side of the system $A \mathbf{D}=\mathbf{f}$ can be interpreted as a vector of weighted means of divided differences, except at the boundaries. Indeed, these weighted means can be expressed as,

$$
\frac{\alpha \delta_{i}+\beta \delta_{i-1}}{\alpha+\beta}
$$

where

$$
\begin{aligned}
& \alpha=\frac{\frac{1}{h_{i}}}{\frac{1}{h_{i-1}}+\frac{1}{h_{i}}}, \\
& \beta=\frac{\frac{1}{h_{i-1}}}{\frac{1}{h_{i-1}}+\frac{1}{h_{i}}} .
\end{aligned}
$$


Now, we can try to replace the expression of the weighted mean in the right hand side of (2.8) by a nonlinear weighted mean, in order to attain adaption to jump discontinuities. Notice that the chosen nonlinear weighted means should penalize large values of $\delta$ 's associated to the presence of discontinuities. We will tackle this task in the next section.

The matrix $A$ of the system $A \mathbf{D}=\mathbf{f}$ in (2.8) satisfies the following result proved in [39] and that will be used in the following sections,

Theorem 2.1. For $1 \leq p \leq \infty$,

$$
\left\|A^{-1}\right\|_{p} \leq \max _{1 \leq i \leq n-1}\left(\frac{1}{h_{i}}+\frac{1}{h_{i+1}}\right)^{-1}
$$

\section{Construction of CUbiC splines ADAPted to Discontinuities: A nONLinear WEIGHTED MEAN}

Now we can design a new limiter that serves our purposes,

$$
\mathrm{HW}_{p}(x, y)= \begin{cases}\frac{\operatorname{sign}(x)+\operatorname{sign}(y)}{2} \frac{\alpha x+\beta y}{\alpha+\beta}\left(1-\left|\beta \frac{x-y}{\alpha x+\beta y}\right|^{p}\right), & |y| \geq|x|,|y|>0, \\ \frac{\operatorname{sign}(x)+\operatorname{sign}(y)}{2} \frac{\alpha x+\beta y}{\alpha+\beta}\left(1-\left|\alpha \frac{x-y}{\alpha x+\beta y}\right|^{p}\right), & |x|>|y|, \\ 0, & x=y=0,\end{cases}
$$

where

$$
\operatorname{sign}(x)= \begin{cases}1, & x>0, \\ -1, & x<0, \\ 0, & x=0 .\end{cases}
$$

The sign function is introduced with the aim of obtaining a bounded value in the case that $x y<0$. As far as we know, it is the first time that this new limiter appears in the literature. These means are a generalization of the means introduced in [33] and reduce to them in the case of a uniform grid spacing.

The new limiter satisfies the properties presented in the following proposition. The proof is trivial and is not included for brevity,

Proposition 3.1. For all $(x, y) \in \mathbb{R}^{2}$, the $\mathrm{HW}_{p}(x, y)$ mean satisfies

(1) $\operatorname{HW}_{p}(x, y)=\operatorname{HW}_{p}(y, x)$.

(2) $\mathrm{HW}_{p}(x, y)=0$, if $x y \leq 0$.

(3) $\mathrm{HW}_{p}(-x,-y)=-\mathrm{HW}_{p}(x, y)$.

Now we can state the following proposition,

Proposition 3.2. The new limiter $\mathrm{HW}_{p}$ verifies the following property. If $x y>0, x=O(1), y=O(1)$, $|x-y|=O(h)$,

$$
\left|\frac{\alpha x+\beta y}{\alpha+\beta}-\operatorname{HW}_{p}(x, y)\right|=O\left(h^{p}\right) .
$$

Proof. Let's suppose without loss of generality that $|y| \geq|x|$. Then, using the definition of $\operatorname{HW}_{p}(x, y)$ given in (3.1), we have that

$$
\begin{aligned}
\left|\frac{\alpha x+\beta y}{\alpha+\beta}-\mathrm{HW}_{p}(x, y)\right| & =\left|\frac{\alpha x+\beta y}{\alpha+\beta}-\frac{\alpha x+\beta y}{\alpha+\beta}\left(1-\left|\beta \frac{x-y}{\alpha x+\beta y}\right|^{p}\right)\right| \\
& =\frac{\alpha x+\beta y}{\alpha+\beta}\left(1-1+\left|\beta \frac{x-y}{\alpha x+\beta y}\right|^{p}\right)=\frac{\alpha x+\beta y}{\alpha+\beta}\left(\left|\beta \frac{x-y}{\alpha x+\beta y}\right|^{p}\right) \\
& =\frac{\alpha x+\beta y}{\alpha+\beta}\left(\left|\frac{\beta}{\alpha+\beta} \frac{\frac{x-y}{\alpha x+\beta y}}{\alpha+\beta}\right|^{p}\right)=O\left(h^{p}\right) .
\end{aligned}
$$


Since we have introduced the new limiter in the right hand side of (2.7), then $x$ and $y$ in (3.1) are neighboring first order divided central differences. If they are computed in a smooth region, we have that $x=O(1)$, $y=O(1)$ and that $\left|\frac{\alpha}{\alpha+\beta}\right|=O(1)$ and $\left|\frac{\beta}{\alpha+\beta}\right|=O(1)$. Then, $\left|\frac{\alpha}{\alpha+\beta} x\right|=O(1),\left|\frac{\beta}{\alpha+\beta} y\right|=O(1),\left|\frac{\alpha x+\beta y}{\alpha+\beta}\right|=O(1)$ and $|x-y|=O(h)$, being $h=\max \left(h_{i}, h_{i+1}\right)$.

Proposition 3.3. The new mean verifies the property,

$$
\left|\mathrm{HW}_{p}(x, y)\right| \leq p \min (|x|,|y|) .
$$

Proof. If $x y<0, \mathrm{HW}_{p}(x, y)=0 \leq p \min (|x|,|y|)$ and the property is satisfied. If $x y>0$, let's suppose again without loss of generality that $|y| \geq|x|$. If we denote by $r=\beta \frac{y-x}{\alpha x+\beta y}$, we can express the absolute value of the new mean as,

$$
\begin{aligned}
\left|\mathrm{HW}_{p}(x, y)\right| & =\left|\frac{\alpha x+\beta y}{\alpha+\beta}\right|\left|\frac{1-r^{p}}{1-r}\right||1-r|=\left|\frac{\alpha x+\beta y}{\alpha+\beta}\right|\left|1+r+r^{2}+\cdots+r^{p-1}\right||1-r| \\
& =\left|\frac{\alpha x+\beta y}{\alpha+\beta}-\frac{\beta}{\alpha+\beta}(y-x)\right|\left|1+r+r^{2}+\cdots+r^{p-1}\right| \\
& =|x|\left|1+r+r^{2}+\cdots+r^{p-1}\right| \leq p|x|,
\end{aligned}
$$

since $|r|<1$.

Proposition 3.4. If $x y>0, \lim _{p \rightarrow \infty} r^{p}=0$, and

$$
\lim _{p \rightarrow \infty} \operatorname{HW}_{p}(x, y)=\frac{\alpha x+\beta y}{\alpha+\beta} .
$$

Proof. Let us suppose that $|y| \geq|x|$. The proof for the other case is carried out in the same way. If $p \rightarrow \infty$, as $r=\frac{\beta}{\alpha+\beta} \frac{y-x}{\frac{\alpha x+\beta y}{\alpha+\beta}}$, then $r^{p} \rightarrow 0$ and, following the same procedure used in the previous proposition to express $\mathrm{HW}_{p}$ in terms of $r$, we get the proof,

$$
\lim _{p \rightarrow \infty} \operatorname{HW}_{p}(x, y)=\lim _{p \rightarrow \infty}\left(\frac{\alpha x+\beta y}{\alpha+\beta}\right)\left(1-r^{p}\right)=\frac{\alpha x+\beta y}{\alpha+\beta} .
$$

With these properties in mind, we can modify the right hand side of the system of equations in (2.7) in order to include the new limiter with the aim of attaining adaption close to discontinuities in the function. Following Proposition 3.2 we choose, $p=3$ in order to maintain $O\left(h^{3}\right)$ accuracy (maximum accuracy depending on the boundary conditions) for the $D_{i}$ and $O\left(h^{4}\right)$ accuracy for the cubic spline at smooth zones. The resulting system of equations is,

$$
\left[\begin{array}{lllllll}
2 & 1 & 0 & 0 & \cdots & 0 & 0 \\
\frac{1}{h_{1}} & 2\left(\frac{1}{h_{1}}+\frac{1}{h_{2}}\right) & \frac{1}{h_{2}} & 0 & \cdots & 0 & 0 \\
0 & \frac{1}{h_{2}} & 2\left(\frac{1}{h_{2}}+\frac{1}{h_{3}}\right) & \frac{1}{h_{3}} & \cdots & 0 & 0 \\
\cdots & \cdots & \cdots & \cdots & \cdots & \cdots & \cdots \\
0 & 0 & 0 & 0 & \cdots & 2\left(\frac{1}{h_{m-1}}+\frac{1}{h_{m}}\right) & \frac{1}{h_{m}} \\
0 & 0 & 0 & 0 & \cdots & 1 & 2
\end{array}\right]\left[\begin{array}{l}
D_{0} \\
D_{1} \\
D_{2} \\
\cdots \\
D_{m-1} \\
D_{m}
\end{array}\right]
$$




$$
=3 \cdot\left[\begin{array}{ll}
\delta_{1} & \\
\left(\frac{1}{h_{1}}+\frac{1}{h_{2}}\right) & \mathrm{HW}_{3}\left(\delta_{1}, \delta_{2}\right) \\
\left(\frac{1}{h_{2}}+\frac{1}{h_{3}}\right) & \mathrm{HW}_{3}\left(\delta_{2}, \delta_{3}\right) \\
\cdots & \\
\left(\frac{1}{h_{m-1}}+\frac{1}{h_{m}}\right) & \mathrm{HW}_{3}\left(\delta_{m-1}, \delta_{m}\right) \\
\delta_{m}
\end{array}\right] .
$$

Since we have introduced the new limiter in the right hand side of (2.7), then $x$ and $y$ in (3.1) are neighboring first order divided central differences. If they are computed in a smooth region, we have that $x=O(1)$, $y=O(1)$ and that $\left|\frac{\alpha}{\alpha+\beta}\right|=O(1)$ and $\left|\frac{\beta}{\alpha+\beta}\right|=O(1)$. Then, $\left|\frac{\alpha}{\alpha+\beta} x\right|=O(1),\left|\frac{\beta}{\alpha+\beta} y\right|=O(1),\left|\frac{\alpha x+\beta y}{\alpha+\beta}\right|=O(1)$ and $|x-y|=O(h)$, being $h=\max \left(h_{i}, h_{i+1}\right)$. Therefore hypothesis of Proposition 3.2 are satisfied.

It is easy to see that the point value discretization described only conserves local information at the sites $x_{i}$. Although it is possible to localize the position of corner discontinuities, there is no hope in localizing the exact position of jumps, as they are lost in the discretization process. Although, the new method presented does not depend on the position of the discontinuity.

Remark 3.5. In the following proofs concerning the accuracy of the proposed reconstruction we will be using the term "full accuracy" meaning fourth order of accuracy for boundary conditions which correspond to true values of the underlying function, and the corresponding lower order in other case. For example it is known that, in general, natural splines provide only second order approximations in the infinity norm.

Theorem 3.6. Let $y(x)$ be a strictly monotone function in $C^{4}([a, b])$. Then, the new cubic splines adapted to discontinuities maintain full order of accuracy.

Proof. Let $y(x)$ be a function in $C^{4}([a, b])$, and $X=\left\{x_{i}\right\}_{i=0}^{m}, x_{0}=a, \quad h_{i}=x_{i}-x_{i-1}$, a partition of $[a, b]$ in subintervals. Let us call

$$
\tilde{s}_{n}(x)=\tilde{g}_{i}(x)=\tilde{a}_{i}\left(x-x_{i}\right)^{3}+\tilde{b}_{i}\left(x-x_{i}\right)^{2}+\tilde{c}_{i}\left(x-x_{i}\right)+\tilde{d}_{i}, x \in\left[x_{i}, x_{i+1}\right],
$$

the adapted cubic spline with the coefficients calculated from (2.3) after solving (3). And in the same way let us consider the classical cubic spline

$$
s_{n}(x)=g_{i}(x)=a_{i}\left(x-x_{i}\right)^{3}+b_{i}\left(x-x_{i}\right)^{2}+c_{i}\left(x-x_{i}\right)+d_{i}, x \in\left[x_{i}, x_{i+1}\right],
$$

with the coefficients given in (2.3), but after solving (2.8). Applying the triangular inequality we have

$$
\left|y(x)-\tilde{s}_{n}(x)\right| \leq\left|y(x)-s_{n}(x)\right|+\left|s_{n}(x)-\tilde{s}_{n}(x)\right| .
$$

Since we know that classical cubic splines are full order accurate, in order to finish the proof it is sufficient to prove that

$$
\left|s_{n}(x)-\tilde{s}_{n}(x)\right|=O\left(h^{4}\right),
$$

where $h=\max _{i} h_{i}$.

From the systems of equations (2.8) and (3) we get that

$$
\|\tilde{D}-D\|_{\infty} \leq\left\|A^{-1}\right\|_{\infty}\|\tilde{\mathbf{f}}-\mathbf{f}\|_{\infty} .
$$

Then applying Theorem 2.1 and Proposition 3.2, we arrive to

$$
\|\tilde{D}-D\|_{\infty} \leq O(h) O\left(h^{2}\right)=O\left(h^{3}\right) .
$$


Now substracting term by term

$$
\left|s_{n}(x)-\tilde{s}_{n}(x)\right| \leq\left|\tilde{a}_{i}-a_{i}\right|\left|x-x_{i}\right|^{3}+\left|\tilde{b}_{i}-b_{i}\right|\left|x-x_{i}\right|^{2}+\left|\tilde{c}_{i}-c_{i}\right|\left|x-x_{i}\right|+\left|\tilde{d}_{i}-d_{i}\right| .
$$

Taking into account the expression of the coefficients of $\tilde{s}_{n}(x)$ and $s_{n}(x)$ given by (2.3) and (3.9) we get

$$
\begin{aligned}
\left|\tilde{d}_{i}-d_{i}\right| & =0, \\
\left|\tilde{c}_{i}-c_{i}\right| & =\left|\tilde{D}_{i}-D_{i}\right|=O\left(h^{3}\right), \\
\left|\tilde{a}_{i}-a_{i}\right| & =\frac{h_{i+1}\left|\tilde{D}_{i+1}-D_{i+1}\right|+\left|\tilde{D}_{i}-D_{i}\right| h_{i+1}}{h_{i+1}^{3}}=O(h), \\
\left|\tilde{b}_{i}-b_{i}\right| & =\frac{h_{i+1}\left|\tilde{D}_{i+1}-D_{i+1}\right|+2\left|\tilde{D}_{i}-D_{i}\right| h_{i+1}}{h_{i+1}^{2}}=O\left(h^{2}\right) .
\end{aligned}
$$

Plugging in (3.10) the expressions in (3.11), we finish the proof.

Remark 3.7. In the previous proof we have not taken into account what happens when the function contains critical points in its domain. We will analyze this case in Section 3.2.

\subsection{Power means}

If we use a uniform grid spacing, the nonlinear weighted means introduced in the previous section transform into de $p$-power means introduced in [33]. The $p$-power mean has the general expression,

$$
H_{p}(x, y)=\frac{\operatorname{sign}(x)+\operatorname{sign}(y)}{2}\left|\frac{x+y}{2}\right|\left(1-\left|\frac{x-y}{x+y}\right|^{p}\right) .
$$

The most important properties that make the power means appropriate are (see $[10,33]$ for more details):

Proposition 3.8. For all $(x, y) \in \mathbb{R}^{2}$, the $H_{p}(x, y)$ mean satisfies

(1) $H_{p}(x, y)=H_{p}(y, x)$.

(2) $H_{p}(x, y)=0$ if $x y \leq 0$.

(3) $H_{p}(-x,-y)=-H_{p}(x, y)$.

(4) $\left|H_{p}(x, y)\right| \leq \max (|x|,|y|)$.

(5) $\left|H_{p}(x, y)\right| \leq p \min (|x|,|y|)$ (related to adaption to the singularities).

(6) If $x=O(1), y=O(1),|y-x|=O(h)$ and $x y>0$ then $\left|\frac{x+y}{2}-H_{p}(x, y)\right|=O\left(h^{p}\right)$.

Assuring the adaption to jump discontinuities of the uniform cubic spline and, at the same time, guaranteeing that the nonlinear modification introduced does not reduce the accuracy at smooth zones, can be done using the $p$-power mean $[10,33]$ with $p=3$ in (3.12). For cubic splines, following property 6 of Proposition 3.8, we have that if $\delta_{j} \cdot \delta_{j+1}>0$,

$$
\left|\frac{\delta_{j}+\delta_{j+1}}{2}-H_{3}\left(\delta_{j}, \delta_{j+1}\right)\right|=O\left(h^{3}\right),
$$

since at smooth zones divided differences $\delta_{j+1}=\delta_{j}=O(1)$.

Using a uniform grid spacing and simplifying and manipulating the right hand side term, the expression of the system of equations in (2.7) transforms into

$$
\left[\begin{array}{lllllll}
2 & 1 & 0 & 0 & \cdots & 0 & 0 \\
1 & 4 & 1 & 0 & \cdots & 0 & 0 \\
0 & 1 & 4 & 1 & \cdots & 0 & 0 \\
\cdots & \cdots & \cdots & \cdots & \cdots & \cdots & \cdots \\
0 & 0 & 0 & 0 & \cdots & 4 & 1 \\
0 & 0 & 0 & 0 & \cdots & 1 & 2
\end{array}\right]\left[\begin{array}{l}
D_{0} \\
D_{1} \\
D_{2} \\
\cdots \\
D_{m-1} \\
D_{m}
\end{array}\right]=6 \cdot\left[\begin{array}{l}
\frac{\delta_{1}}{2} \\
\frac{\delta_{2}+\delta_{1}}{2} \\
\frac{\delta_{3}+\delta_{2}}{2} \\
\cdots \\
\frac{\delta_{m}+\delta_{m-1}}{2} \\
\frac{\delta_{m}}{2}
\end{array}\right]
$$


Replacing the arithmetic mean in (3.14) by the $p$-power mean in (3.12) with $p=3$, we obtain the system of equations for the adapted spline,

$$
\left[\begin{array}{lllllll}
2 & 1 & 0 & 0 & \cdots & 0 & 0 \\
1 & 4 & 1 & 0 & \cdots & 0 & 0 \\
0 & 1 & 4 & 1 & \cdots & 0 & 0 \\
\cdots & \cdots & \cdots & \cdots & \cdots & \cdots & \cdots \\
0 & 0 & 0 & 0 & \cdots & 4 & 1 \\
0 & 0 & 0 & 0 & \cdots & 1 & 2
\end{array}\right]\left[\begin{array}{l}
D_{0} \\
D_{1} \\
D_{2} \\
\cdots \\
D_{m-1} \\
D_{m}
\end{array}\right]=6 \cdot\left[\begin{array}{l}
\frac{\delta_{1}}{2} \\
H_{3}\left(\delta_{2}, \delta_{1}\right) \\
H_{3}\left(\delta_{3}, \delta_{2}\right) \\
\cdots \\
H_{3}\left(\delta_{m}, \delta_{m-1}\right) \\
\frac{\delta_{m}}{2}
\end{array}\right]
$$

Remark 3.9. The process followed in this subsection to adapt the splines in a uniform grid-spacing to the presence of jump discontinuities in the function can be perfectly used for parametrized splines. We can parametrize the spline in (2.1) just expressing it in terms of another variable $u$ that varies in the interval $[0,1]$ for every polynomial segment of the spline, with

$$
u=\frac{x-x_{i}}{x_{i+1}-x_{i}} .
$$

With this parametrization, the grid-spacing can be interpreted to be always $h=1$ and the divided differences transform into differences. This means that the new technique can be used even when the original data does not come from the sampling of a function and, for example, we can construct closed curves very easily.

\subsection{Assuring maximum accuracy for nonlinear means close to critical points}

Property 2 of Propositions 3.1 and 3.8 imply that the use of the new mean (3.1) or the power mean (3.12) produces order reduction when the arguments have different sign and property 6 of Propositions 3.2 and 3.8 do not allow to maintain the approximation order when one of the arguments is $O(h)$. Both conditions happen at singular points or close to them. This problem can be solved translating both arguments, obtaining the mean and then translating back the result by the same amount. The problem arises when deciding how many points around those critical points must be translated in order to assure optimal accuracy. Mind that close to critical points both arguments are $O(h)$. Let us introduce the definition of the translation $T$.

Definition 3.10. Given $h>0$, a translation $T$ is any function $T: \mathbb{R}^{2} \rightarrow \mathbb{R}$ satisfying

(1) $T(0,0)=0$.

(2) $T(x, y)=T(y, x)$.

(3) $\operatorname{sign}(x+T(x, y)) \operatorname{sign}(y+T(x, y))>0, \forall(x, y) \neq(0,0)$.

(4) If $(x, y) \neq(0,0)$, with $|x| \leq|y|$, then $\operatorname{sign}(x+T(x, y)) \operatorname{sign}(y)>0$.

(5) $\min \{|x+T(x, y)|,|y+T(x, y)|\}=O(1), \forall(x, y) \neq(0,0)$, with $\min \{|x|,|y|\}=O\left(h^{\alpha}\right)$, for some $\alpha \leq 0$.

Remark 3.11. Property 3 of Definition 3.10 ensures that it is going to be always possible to compute expression (3.1) without problems in the denominator. Property 4 assures a translation towards the largest of the arguments in absolute value. And property 5 is essential to keep the reconstruction order (see Props. 3.2 and 3.13), and the adaption in case of discontinuities (see Props. 3.3 and 3.14).

The nonlinear weighted mean in (3.1) transforms into

$$
\operatorname{HWT}_{p}(x, y):=\operatorname{HW}_{p}(x+T, y+T)-T
$$

with the translation. It is easy to prove the same kind of results as in Propositions 3.1-3.3 with this new mean. In particular,

Proposition 3.12. For all $(x, y) \in \mathbb{R}^{2}$, the $\operatorname{HWT}_{p}(x, y)$ mean satisfies 
(1) $\operatorname{HWT}_{p}(x, y)=\operatorname{HWT}_{p}(y, x)$.

(2) $\operatorname{HWT}_{p}(-x,-y)=-\mathrm{HWT}_{p}(x, y)$.

Proposition 3.13. The new limiter $\mathrm{HWT}_{p}$ verifies the following property,

$$
\left|\frac{\alpha x+\beta y}{\alpha+\beta}-\operatorname{HWT}_{p}(x, y)\right|=O\left(h^{p}\right) .
$$

The previous three properties are trivial to prove and are not included for brevity.

Given $|x| \leq|y|$, the authors propose in [7] a translation that is $T=2|x|$ at the places where there is a change of sign in the arguments of the mean. This translation does not satisfy property 5 in Definition 3.10, and in fact it does not guarantee the reconstruction order close to the critical points. Instead, it seems more suitable to use a proper translation in the sense of Definition 3.10 defined as,

$$
T= \begin{cases}\operatorname{sign}(y)(|x|+\epsilon), & \text { if } x y<0, \\ \operatorname{sign}(y) \epsilon, & \text { if } x y \geq 0\end{cases}
$$

with $\epsilon>0, \epsilon=O(1) . \epsilon$ can be chosen much smaller $\epsilon=O\left(h^{p}\right), p \geq 1$ and it is a better option when $\min \{|x|,|y|\}=$ $O(1)$. With this translation it is easy to prove the following proposition.

Proposition 3.14. With the translation defined in (3.18) and assuming, without loss of generality, that $|x| \leq$ $|y|$, the nonlinear weighted mean in (3.16) is bounded by

$$
\left|\operatorname{HWT}_{p}(x, y)\right| \leq p|x|+(p+1) \epsilon .
$$

Proof. We will consider five different cases to get the proof:

- If $x=0, y=0$, then $T=0$ and $\operatorname{HWT}_{p}(x, y)=0$ and the result is trivially true.

- If $x \leq 0$ and $y>0$, the translation has the expression $T=-x+\epsilon>0$ and the weighted mean is bounded by,

$$
\epsilon \leq \mathrm{HW}_{p}(\epsilon, y-x+\epsilon) \leq p \epsilon,
$$

due to Proposition 3.3 and the fact that $\mathrm{HW}_{p}$ is a mean. Now the resultant value of the mean can be positive or negative:

- If $\operatorname{HWT}_{p}(x, y)=\mathrm{HW}_{p}(x+T, y+T)-T=\mathrm{HW}_{p}(\epsilon, y-x+\epsilon)+x-\epsilon \geq 0$, then

$$
\left|\operatorname{HWT}_{p}(x, y)\right| \leq p \epsilon+|x|-\epsilon=|x|+(p-1) \epsilon .
$$

- If $\operatorname{HWT}_{p}(x, y)=\mathrm{HW}_{p}(x+T, y+T)-T=\mathrm{HW}_{p}(\epsilon, y-x+\epsilon)+x-\epsilon<0$, then

$$
\left|\operatorname{HWT}_{p}(x, y)\right|=-x+\epsilon-\mathrm{HW}_{p}(\epsilon, y-x+\epsilon) \leq|x| .
$$

- If $x \geq 0$ and $y<0$, the translation takes the value $T=-x-\epsilon<0$ and the weighted mean is bounded by,

$$
-p \epsilon \leq \operatorname{HW}_{p}(-\epsilon, y-x-\epsilon) \leq-\epsilon,
$$

due again to Proposition 3.3 and the fact that $\mathrm{HW}_{p}$ is a mean. Now the resultant value of the mean can be positive or negative:

- If $\operatorname{HWT}_{p}(x, y)=\mathrm{HW}_{p}(x+T, y+T)-T=\mathrm{HW}_{p}(-\epsilon, y-x-\epsilon)+x+\epsilon \geq 0$, then

$$
\left|\operatorname{HWT}_{p}(x, y)\right| \leq|x| \text {. }
$$

- If $\operatorname{HWT}_{p}(x, y)=\mathrm{HW}_{p}(x+T, y+T)-T=\mathrm{HW}_{p}(-\epsilon, y-x-\epsilon)+x+\epsilon<0$, then

$$
\left|\operatorname{HWT}_{p}(x, y)\right| \leq|x|+(p+1) \epsilon .
$$


- If $x>0$ and $y>0$, the translation takes the value $T=\epsilon>0$. $\operatorname{Then}_{H_{W W T}}(x, y)=\operatorname{HW}_{p}(x+T, y+T)-T=$ $\mathrm{HW}_{p}(x+\epsilon, y+\epsilon)-\epsilon>0$ and the weighted mean is bounded by,

$$
x+\epsilon \leq \mathrm{HW}_{p}(x+\epsilon, y+\epsilon) \leq p(x+\epsilon) .
$$

In this case $\mathrm{HW}_{p}(x+\epsilon, y+\epsilon) \geq 0$ and

$$
\left|\operatorname{HWT}_{p}(x, y)\right| \leq p|x|+(p-1) \epsilon .
$$

- If $x<0$ and $y<0$, the translation takes the value $T=-\epsilon<0$. Then $\operatorname{HWT}_{p}(x, y)=\operatorname{HW}_{p}(x+T, y+T)-T=$ $\mathrm{HW}_{p}(x-\epsilon, y-\epsilon)+\epsilon<0$ and the weighted mean is bounded by,

$$
p(x-\epsilon) \leq \mathrm{HW}_{p}(x-\epsilon, y-\epsilon) \leq x-\epsilon .
$$

In this case $\mathrm{HW}_{p}(x-\epsilon, y-\epsilon)<0$ and

$$
\left|\operatorname{HWT}_{p}(x, y)\right|=-\mathrm{HW}_{p}(x-\epsilon, y-\epsilon)-\epsilon \leq-p x+p \epsilon-\epsilon=p|x|+(p-1) \epsilon .
$$

With the information provided by Proposition 3.14, it seems logical to suggest a nonlinear $\epsilon$ that is adapted to the presence of critical points in the sense that is $O(1)$ at critical points (and around them) and that goes to zero away from them. The objective is that the translation does not affect the adaption attained at jump discontinuities (provided by Prop. 3.3 for the non-translated mean) unless a change of sign in the first derivative is placed exactly at the discontinuity. Inspired by the smoothness indicators proposed in [35], we propose an $\epsilon$ with the following expression,

$$
\epsilon_{j}:=\tau \mu \tilde{\epsilon}_{j},
$$

and

$$
\tilde{\epsilon}_{j}:=\frac{1}{\left(\left|\mathrm{IS}_{j}\right|+\xi\right)^{t}},
$$

where $\mathrm{IS}_{j}$ is a smoothness indicator. The idea is that these indicators are large when a jump discontinuity affects the stencil used to obtain it, and small otherwise. We choose the parameter $\mu=h^{4}$. This normalization is performed to assure that the maximum $\epsilon_{j}$ takes an order given by the parameter $\tau$ around critical points. In the numerical experiments, a reasonable value for this parameter is $\tau \in(0,1]$ with $\tau=O(1)$. We have chosen $\tau=1$ in our experiments. The parameter $\xi=h^{4}$ is included to avoid divisions by zero. The parameter $t$ modifies the rate of convergence of the function in (3.22) towards zero. We have chosen $t=1$ in our experiments.

As mentioned before, $\mathrm{IS}_{j}$ is a smoothness indicator. In [35], Jiang and Shu propose to obtain smoothness indicators using something similar to the total variation, but based in the $L^{2}$ norm, so that the result is smoother than the total variation. The proposed formula is just a scaled sum of the $L^{2}$ norms of the derivatives of the interpolation polynomials in the cell-averages $p_{j}(x)$ over the interval $\left(x_{j-1 / 2}, x_{j+1 / 2}\right)$. The expression proposed for a uniform grid spacing is,

$$
\mathrm{IS}_{j}=\sum_{l=1}^{r-1} h^{2 l-1} \int_{x_{j-\frac{1}{2}}}^{x_{j+\frac{1}{2}}}\left(\frac{d^{l}}{\mathrm{~d} x^{l}} p_{j}(x)\right)^{2} \mathrm{~d} x,
$$

where $r$ is the degree of the polynomial $p_{j}(x)$. The scaling factor $h^{2 l-1}=\Delta x^{2 l-1}$ is introduced to assure that the final formulae for the smoothness indicator do not depend on the mesh size $h=\Delta x$. We have chosen the smoothness indicators in the point values for $r=2$ that result from (3.23). They have the following expression for a uniform grid-spacing,

$$
\begin{aligned}
\mathrm{IS}_{j} & =\frac{13}{12}\left(f_{j-1}-2 f_{j}+f_{j+1}\right)^{2}+(1 / 14)\left(f_{j-1}-4 f_{j}+3 f_{j+1}\right)^{2}, \\
\mathrm{IS}_{j+1} & =\frac{13}{12}\left(f_{j}-2 f_{j+1}+f_{j+2}\right)^{2}+(1 / 14)\left(f_{j+2}-f_{j}\right)^{2} .
\end{aligned}
$$


For these smoothness indicators, the polynomial $p_{j}(x)$ is a parabola. We could have chosen a smoothness indicator with a wider stencil but the results are similar and more expensive computationally.

One important property of these smoothness indicators is the one presented in the following proposition.

Proposition 3.15. The smoothness indicators presented in (3.24) and (3.25) verify that

$$
\begin{aligned}
\mathrm{IS}_{j} & =\left(h f_{j+1}^{\prime}\right)^{2}\left(1+O\left(h^{2}\right)\right), \\
\mathrm{IS}_{j+1} & =\left(h f_{j+1}^{\prime}\right)^{2}\left(1+O\left(h^{2}\right)\right),
\end{aligned}
$$

at smooth zones.

The proof of this property is straightforward and it is not included for brevity. The interested reader can refer to [3] for a detailed description of the process for different smoothness indicators. If we develop $f_{j}$ and $f_{j-1}$ using Taylor expansion around $x_{j+1}$ and replace their expressions in (3.24) and (3.25) we get the proof. The property is important, as it assures that $\epsilon_{j}$ is small away from critical points and large close to them. Moreover, $\mathrm{IS}_{j}$ is $O(1)$ at a jump discontinuity following (3.24) and (3.25). Thus (3.21) assures that $\epsilon_{j}=O\left(h^{4}\right)$ at a jump discontinuity, so the translation in (3.18) would be given by $T=\operatorname{sign}(y)\left(|x|+O\left(h^{4}\right)\right)$. Following (3.19), if $|x| \leq|y|$, the mean would be bounded by,

$$
\left|\operatorname{HWT}_{p}(x, y)\right|<p|x|+(p+1) O\left(h^{4}\right),
$$

and it would not affect the adaption properties of the nonlinear weighted mean, conserving in practice the property exposed in Proposition 3.3 for the non-translated mean. At smooth zones, the translation defined using (3.21) ensures to attain full accuracy even around critical points, since $\epsilon_{j}=O(1)$ in this case, and allows the application of Proposition 3.2. In order to measure the smoothness of our function, we only need one smoothness indicator, so we choose for example (3.24).

Remark 3.16. For a non uniform grid spacing, it is still possible to obtain a smoothness indicator like the ones in (3.24) and (3.25) using (3.23). In this case, it is not possible to get rid of the dependency of the grid spacing and the closed expressions are not as simple as in the uniform case.

Remark 3.17. In the numerical experiments presented for the uniform grid spacing, the nonlinear translated mean (3.16) with the translation defined in (3.18) and the $\epsilon$ defined in (3.21) has been used.

With the introduction of the $\mathrm{HWT}_{p}$ means we can give the following two theorems, which generalize the result in Theorem 3.6.

Theorem 3.18. Let $y(x)$ be a function in $C^{4}([a, b])$. Then, the new cubic splines adapted to discontinuities based on the nonlinear mean $\mathrm{HWT}_{p}$ defined in (3.16) through the translation $T$ defined in (3.18) maintain full order of accuracy.

Proof. The proof is basically the same as for Theorem 3.6. The only difference is the application of Proposition 3.13 instead of Proposition 3.2.

Let $A^{-1}=\left(\alpha_{i, j}\right)$ be the inverse of the coefficient matrix of system (3.15). There is a clear dependence between the grid spacing and the size of the matrix, and it is not so clear but also between the size of the matrix and the entries of $A^{-1}$. In fact, using basic algebra and numerical analysis next lemma can be proven,

Lemma 3.19. Let $A^{-1}=\left(\alpha_{i, j}\right)$ be the inverse of the coefficient matrix of system (3.15). Then,

$$
\alpha_{i, i \pm s}=O\left(h^{\gamma s}\right), \quad s \geq 0, \quad i \in\{1, \ldots, m\}, \quad i \pm s=1, \ldots, m,
$$

with $\gamma=\log _{2}(2+\sqrt{3})$. 
Proof. The first observation is that according to an adaption to our case of the general result for tridiagonal matrices in [38], the entries of $A^{-1}$ can be computed as

$$
\alpha_{i, j}= \begin{cases}(-1)^{i+j \frac{\phi(2, i-1,4) \phi(4, m-j, 2)}{\phi(2, m, 2)},}, & i<j, \\ \frac{\phi(2, i-1,4) \phi(4, m-i, 2)}{\phi(2, m, 2)}, & i=j, \\ (-1)^{i+j \frac{\phi(2, j-1,4) \phi(4, m-i, 2)}{\phi(2, m, 2)},}, & i>j,\end{cases}
$$

where $\phi_{k}:=\phi\left(d_{1}, k, d_{k}\right)$, is the solution of the recurrence equation

$$
\begin{aligned}
\phi_{0} & =1, \phi_{1}=d_{1}, \\
\phi_{i} & =4 \phi_{i-1}-\phi_{i-2}, \quad i=2, \ldots, k-1, \\
\phi_{k} & =d_{k} \phi_{k-1}-\phi_{k-2} .
\end{aligned}
$$

Using theory about linear finite difference equations one can give the general term of $\phi_{k}$ in terms of the roots of the characteristic polynomial $\lambda^{2}-4 \lambda+1=0$. From Theorem 2.1 we deduce that $\lim _{h \rightarrow 0} \alpha_{i, j}=0, \forall i, j=$ $1, \ldots, m$. Therefore we can assume that $\alpha_{i, j}=O\left(h^{\beta}\right)$, for some value of $\beta \geq 0$. We are going to compute $\beta$ for each $i, j=1, \ldots, m$. In order to do so we consider two grid spacings $h$ and $\frac{h}{2}$, that is, sizes $m \times m$ and $(2 m-1) \times(2 m-1)$ of the matrix $A$. Let us call $\alpha_{i, j}$ the entries of the inverse of the matrix $A$ of order $m$ and $\mu_{i, j}$ the entries of the inverse of the matrix $A$ of order $2 m-1$. If we compute the $\operatorname{limit} \lim _{m \rightarrow \infty} \frac{\alpha_{i, j}}{\mu_{2 i-1,2 j-1}}$, and is equal to a constant $M$, then $\beta=\log _{2}(M)$.

Thus, we consider for $s \geq 0$,

$$
\begin{aligned}
\lim _{m \rightarrow \infty}\left|\frac{\alpha_{i, i-s}}{\mu_{2 i-1,2 i-2 s-1}}\right| & =\lim _{m \rightarrow \infty} \frac{\frac{\phi(2, i-s-1,4) \phi(4, m-i, 2)}{\phi(2, m, 2)}}{\frac{\phi(2,2 i-2 s-2,4) \phi(4,2 m-2 i+1,2)}{\phi(2,2 m-1,2)}} \\
& =\lim _{m \rightarrow \infty} \frac{\frac{(2+\sqrt{3})^{i-s}(2+\sqrt{3})^{m-i-2}}{(2+\sqrt{3})^{m-2}}}{\frac{(2+\sqrt{3})^{2 i-2 s-2}(2+\sqrt{3})^{2 m-2 i-1}}{(2+\sqrt{3})^{2 m-3}}}=(2+\sqrt{3})^{s} .
\end{aligned}
$$

From (3.31) we can apply logarithms and symmetry to get the general result $\alpha_{i, i \pm s}=O\left(h^{\gamma s}\right), s \geq 0, i \in$ $\{1, \ldots, m\}, i \pm s=1, \ldots, m$, with $\gamma=\log _{2}(2+\sqrt{3})$.

We can give now the following theorem about the approximation order of the presented nonlinear cubic splines,

Theorem 3.20. Let $y(x)$ be a function in $C^{4}([a, b] \backslash D)$, where $D$ is a finite set of isolated jump discontinuities. Let $X=\left\{x_{j}\right\}_{j=0}^{m}$ be a grid in $[a, b]$. Then, the new cubic splines adapted to discontinuities based on the nonlinear mean $\mathrm{HWT}_{p}$ defined in (3.16) through the translation $\mathrm{T}$ defined in (3.18) maintain full order of accuracy at smooth zones away from the isolated discontinuities.

Proof. We carry out the proof for the uniform case because of simplicity, although the same arguments are valid for the nonuniform case. Let us suppose without loss of generality that the initial data come from a $C^{4}([a, b] \backslash\{\mu\})$ function $y(x)$ with an isolated jump discontinuity located at $\mu$ in the interval $\left[x_{i}, x_{i+1}\right]$ for some $i \in\{2, \ldots, m-3\}$. We are going to prove that the nonlinear cubic splines attain full order accuracy in $\left[a, x_{i-2}\right]$. By symmetry the result will also hold for $\left[x_{i+3}, b\right]$. Let us call $g_{[a, b]}$ the classical cubic spline with original data, $\hat{g}_{[a, b]}$ the corresponding nonlinear cubic spline in the hypothesis of the theorem, and $\tilde{g}_{[a, b]}$ an auxiliary classical cubic spline built with certain modified data. The proof is based on the following four points, which will be proved in detail later on: 
(1) If we modified the entries $\hat{f}_{i}, \hat{f}_{i+1}$ of the vector $\hat{f}$ at the right hand side of system $A \hat{D}=\hat{f}$ in (3.15) by a quantity of order $O(1)$, then we get another system $A \tilde{D}=\tilde{f}$, such that $\left|\hat{D}_{i+j}-\tilde{D}_{i+j}\right|=O\left(h^{\gamma(j-1)}\right), j \geq 1$ and $\left|\hat{D}_{i-j}-\tilde{D}_{i-j}\right|=O\left(h^{\gamma j}\right), j \geq 0$, with $\gamma=\log _{2}(2+\sqrt{3})$. Therefore the modification gives an associated reconstruction $\tilde{g}_{[a, b]}$ which satisfies $\left|\hat{g}_{[a, b]}(x)-\tilde{g}_{[a, b]}(x)\right|=O\left(h^{4}\right)$ in $\left[a, x_{i-2}\right]$.

(2) We can find a modification of the type described in point 1 such that the right hand side of system (3.15) plus this modification can be seen as the right hand side of the system (3.14) for linear cubic splines with modified data $\tilde{y}_{j}$ free of any discontinuity, i.e. coming from an underlying $C^{4}([a, b])$ function $\tilde{y}(x)$.

(3) If a linear cubic spline $g_{[a, b]}$ attains full order of approximation in $[a, b]$, then also the function given by the linear cubic spline $g_{\left[a, x_{i-2}\right]}$ attains full order of approximation in $\left[a, x_{i-2}\right]$.

(4) The difference between the original data $y_{j}, j=0, \ldots, i-2$ and the modified data $\tilde{y}_{j}$ of step 2 is fourth order. And therefore the difference between $g_{\left[a, x_{i-2}\right]}$ and $\tilde{g}_{\left[a, x_{i-2}\right]}$ is also fourth order accurate.

If we assume the points $1-4$ proved, then it is immediate that for any $x \in\left[a, x_{i-2}\right]$,

- $\left|\hat{g}_{[a, b]}(x)-\tilde{g}_{[a, b]}(x)\right|=O\left(h^{4}\right)$ due to point 1 .

- $\left|\tilde{g}_{[a, b]}(x)-\tilde{y}(x)\right|=O\left(h^{p}\right)$ where $p \leq 4$ is the full accuracy attained by the considered linear cubic spline (depends on the initial boundary conditions) due to point 2 .

- $\left|\tilde{y}(x)-\tilde{g}_{\left[a, x_{i-2}\right]}(x)\right|=O\left(h^{p}\right)$, due to point 3 .

- $\left|\tilde{g}_{\left[a, x_{i-2}\right]}(x)-g_{\left[a, x_{i-2}\right]}(x)\right|=O\left(h^{4}\right)$ due to point 4 .

- $\left|g_{\left[a, x_{i-2}\right]}(x)-y(x)\right|=O\left(h^{p}\right)$ due to point 3 .

And thus

$$
\begin{aligned}
\left|\hat{g}_{[a, b]}(x)-y(x)\right| \leq & \left|\hat{g}_{[a, b]}(x)-\tilde{g}_{[a, b]}(x)\right|+\left|\tilde{g}_{[a, b]}(x)-\tilde{y}(x)\right| \\
& +\left|\tilde{y}(x)-\tilde{g}_{\left[a, x_{i-2}\right]}(x)\right|+\left|\tilde{g}_{\left[a, x_{i-2}\right]}(x)-g_{\left[a, x_{i-2}\right]}(x)\right| \\
& +\left|g_{\left[a, x_{i-2}\right]}(x)-y(x)\right|=O\left(h^{4}\right)+O\left(h^{p}\right)+O\left(h^{p}\right)+O\left(h^{4}\right)+O\left(h^{p}\right) \\
= & O\left(h^{p}\right), \quad \forall x \in\left[a, x_{i-2}\right],
\end{aligned}
$$

what will finish the proof.

Therefore, we need to prove the points $1-4$. Let us start with point 1.

(1) Let us consider the system,

$$
\left[\begin{array}{lllllll}
2 & 1 & 0 & 0 & \cdots & 0 & 0 \\
1 & 4 & 1 & 0 & \cdots & 0 & 0 \\
\cdots & \cdots & \cdots & \cdots & \cdots & \cdots & \cdots \\
0 & 1 & 4 & 1 & \cdots & 0 & 0 \\
0 & 1 & 4 & 1 & \cdots & 0 & 0 \\
\cdots & \cdots & \cdots & \cdots & \cdots & \cdots & \cdots \\
0 & 0 & 0 & 0 & \cdots & 4 & 1 \\
0 & 0 & 0 & 0 & \cdots & 1 & 2
\end{array}\right]\left[\begin{array}{l}
\tilde{D}_{0} \\
\tilde{D}_{1} \\
\cdots \\
\tilde{D}_{i} \\
\tilde{D}_{i+1} \\
\cdots \\
\tilde{D}_{m-1} \\
\tilde{D}_{m}
\end{array}\right]=6\left[\begin{array}{l}
\frac{\delta_{1}}{2} \\
H_{3}\left(\delta_{1}, \delta_{2}\right) \\
\cdots \\
H_{3}\left(\delta_{i}, \delta_{i+1}\right) \\
H_{3}\left(\delta_{i+1}, \delta_{i+2}\right) \\
\cdots \\
H_{3}\left(\delta_{m-1}, \delta_{m}\right) \\
\frac{\delta_{m}}{2}
\end{array}\right]+\left[\begin{array}{l}
0 \\
0 \\
\cdots \\
0(1) \\
0(1) \\
\cdots \\
0 \\
0
\end{array}\right]
$$

Then the difference $\left|\hat{D}_{i \pm j}-\tilde{D}_{i \pm j}\right|, i \pm j=0, \ldots, m$, between the solution of the system $A \hat{D}=\hat{f}$ in (3.15) and $A \tilde{D}=\tilde{f}$ in $(3.33)$ is given by $\hat{D}-\tilde{D}=A^{-1}(f-\tilde{f})$. Let us compute a concrete $\left|\hat{D}_{i \pm j}-\tilde{D}_{i \pm j}\right|$ for a fixed value of $j$,

$$
\left|\hat{D}_{i \pm j}-\tilde{D}_{i \pm j}\right|=\sum_{s=0}^{m} \alpha_{i \pm j, s}\left(f_{s}-\tilde{f}_{s}\right)=\alpha_{i \pm j, i} O(1)+\alpha_{i \pm j, i+1} O(1) .
$$


From Lemma 3.19 we get the estimation for the needed entries of the inverse $A^{-1}$, given by $\alpha_{i \pm j, i}=O\left(h^{\gamma j}\right)$ and $\alpha_{i \pm j, i+1}=O\left(h^{\gamma|1 \mp j|}\right)$, with $\gamma=\log _{2}(2+\sqrt{3})$. Thus,

$$
\left|\hat{D}_{i \pm j}-\tilde{D}_{i \pm j}\right|=O\left(h^{\gamma j}\right) O(1)+O\left(h^{\gamma|1 \mp j|}\right) O(1),
$$

and therefore $\left|\hat{D}_{i+j}-\tilde{D}_{i+j}\right|=O\left(h^{\gamma(j-1)}\right), j \geq 1$ and $\left|\hat{D}_{i-j}-\tilde{D}_{i-j}\right|=O\left(h^{\gamma j}\right), j \geq 0$.

Using now expressions (2.3) we get,

$$
\left|\hat{g}_{[a, b]}(x)-\tilde{g}_{[a, b]}(x)\right|= \begin{cases}O\left(h^{4}\right) & \text { if } x \in\left[x_{i-j}, x_{i-j+1}\right], j \geq 3, \\ O\left(h^{\gamma+2}\right) & \text { if } x \in\left[x_{i-2}, x_{i-1}\right], \\ O(h) & \text { if } x \in\left[x_{i-1}, x_{i}\right] \\ O(h) & \text { if } x \in\left[x_{i}, x_{i+1}\right] \\ O(h) & \text { if } x \in\left[x_{i+1}, x_{i+2}\right], \\ O\left(h^{\gamma+2}\right) & \text { if } x \in\left[x_{i+2}, x_{i+3}\right], \\ O\left(h^{4}\right) & \text { if } x \in\left[x_{i+j}, x_{i+j+1}\right], j \geq 3 .\end{cases}
$$

(2) From the right hand sides of (3.14) and (3.15) we impose the following system of equations based on data to the left of the interval affected by the discontinuity,

$$
\left[\begin{array}{l}
\delta_{1} \\
2 H_{3}\left(\delta_{1}, \delta_{2}\right) \\
\cdots \\
2 H_{3}\left(\delta_{i-2}, \delta_{i-1}\right)
\end{array}\right]=\left[\begin{array}{l}
\tilde{\delta}_{1} \\
\tilde{\delta}_{1}+\tilde{\delta}_{2} \\
\cdots \\
\tilde{\delta}_{i-2}+\tilde{\delta}_{i-1}
\end{array}\right],
$$

where $\tilde{\delta}_{j}:=\frac{\tilde{y}_{j}-\tilde{y}_{j-1}}{h}$ stands for the first order differences based on modified input data $y_{j}$. Solving the system (3.34) we get $\tilde{\delta}_{1}=\delta_{1}, \tilde{\delta}_{j}=2 H_{3}\left(\delta_{j-1}, \delta_{j}\right)-\tilde{\delta}_{j-1}, j=2, \ldots, i-1$. Let us now prove by induction that $\tilde{\delta}_{j}-\delta_{j}=O\left(h^{3}\right), j=1, \ldots, i-1$. It is trivially true for $j=1$ since $\tilde{\delta}_{1}=\delta_{1}$. Let us suppose then by hypothesis of induction that $\tilde{\delta}_{j}-\delta_{j}=O\left(h^{3}\right)$. For $j+1$ we have,

$$
\begin{aligned}
\tilde{\delta}_{j+1}-\delta_{j+1} & =2 H_{3}\left(\delta_{j}, \delta_{j+1}\right)-\tilde{\delta}_{j}-\delta_{j+1}=2 H_{3}\left(\delta_{j}, \delta_{j+1}\right)-\delta_{j}-\delta_{j+1}+O\left(h^{3}\right) \\
& =2\left(H_{3}\left(\delta_{j}, \delta_{j+1}\right)-\frac{\delta_{j}+\delta_{j+1}}{2}\right)+O\left(h^{3}\right)=O\left(h^{3}\right) .
\end{aligned}
$$

Now, from the values of $\tilde{\delta_{j}}$ it is immediate to compute the corresponding values $\tilde{y}_{j}$ starting from $\tilde{y}_{0}=y_{0}$. In the same way from the system,

$$
\left[\begin{array}{l}
2 H_{3}\left(\delta_{i+2}, \delta_{i+3}\right) \\
2 H_{3}\left(\delta_{i+3}, \delta_{i+4}\right) \\
\cdots \\
\delta_{m}
\end{array}\right]=\left[\begin{array}{l}
\tilde{\delta}_{i+2}+\tilde{\delta}_{i+3} \\
\tilde{\delta}_{i+3}+\tilde{\delta}_{i+4} \\
\cdots \\
\tilde{\delta}_{m}
\end{array}\right],
$$

we obtain the values for $\tilde{\delta}_{j}, j=i+2, \ldots, m$ satisfying

$$
\tilde{\delta}_{j}-\delta_{j}=O\left(h^{3}\right), \quad j=i+2, \ldots, m .
$$

From these values $\tilde{\delta}_{j}, j=i+2, \ldots, m$, it is easy again to obtain corresponding values for $\tilde{y}_{j}$.

Now, we build a $C^{4}$ function in $[a, b]$, just by using piecewise Hermite interpolation $q_{j}(x)$ of the required degree at each interval $\left[x_{j}, x_{j+1}\right], j=1, \ldots, i-2,\left[x_{i-1}, x_{i+1}\right]$, and $\left[x_{j}, x_{j+1}\right], j=i+1, \ldots, m-1$. At a interval $\left[x_{j}, x_{j+1}\right]$ we use $\left\{y_{j}, y_{j}^{(i)}, y_{j}^{(i i)}, y_{j}^{(i i i)}, y_{j}^{(i v)}\right\}$ and $\left\{y_{j+1}, y_{j+1}^{(i)}, y_{j+1}^{(i i)}, y_{j+1}^{(i i i)}, y_{j+1}^{(i v)}\right\}$ as initial conditions to build the piecewise Hermite intepolation. We observe then, that due to the use of these conditions coming from a smooth original function, we ensure that the derivative that appear in the error formula for cubic 
linear spline $g_{\left[a, x_{i-2}\right]}$ is bounded independently of the grid size. We then consider $\tilde{y}_{i}=q_{i-1}\left(x_{i}\right)$. We notice that $H\left(\delta_{i}, \delta_{i+1}\right)-\frac{\tilde{\delta}_{i}+\tilde{\delta}_{i+1}}{2}=O(1), H\left(\delta_{i+1}, \delta_{i+2}\right)-\frac{\tilde{\delta}_{i+1}+\tilde{\delta}_{i+2}}{2}=O(1)$, and therefore using also (3.35) and (3.37) we have

$$
\left[\begin{array}{l}
\frac{\delta_{1}}{2} \\
H_{3}\left(\delta_{1}, \delta_{2}\right) \\
\cdots \\
H_{3}\left(\delta_{i}, \delta_{i+1}\right) \\
H_{3}\left(\delta_{i+1}, \delta_{i+2}\right) \\
\cdots \\
H_{3}\left(\delta_{m-1}, \delta_{m}\right) \\
\frac{\delta_{m}}{2}
\end{array}\right]-\left[\begin{array}{l}
\frac{\tilde{\delta}_{1}}{2} \\
\frac{\tilde{\delta}_{1}+\tilde{\delta}_{2}}{2} \\
\cdots \\
\frac{\tilde{\delta}_{i}+\tilde{\delta}_{i+1}}{2} \\
\frac{\tilde{\delta}_{i+1}+\tilde{\delta}_{i+2}}{2} \\
\cdots \\
\frac{\tilde{\delta}_{m-1}+\tilde{\delta}_{m}}{2} \\
\frac{\tilde{\delta}_{m}}{2}
\end{array}\right]=\left[\begin{array}{l}
0 \\
0 \\
\cdots \\
0(1) \\
0(1) \\
\cdots \\
0 \\
0
\end{array}\right] .
$$

(3) If the hypothesis needed to guarantee full order of approximation are satisfied in the whole interval $[a, b]$, then these same hypothesis are also satisfied in the subinterval $\left[a, x_{i-2}\right]$. Therefore point 3 is proved.

(4) The first affirmation in point 4 was already proved in point 2 since $\delta_{j}-\tilde{\delta}_{j}=O\left(h^{3}\right), j=1, \ldots, i-2, \delta_{1}=\tilde{\delta}_{1}$ directly implies $y_{j}-\tilde{y}_{j}=O\left(h^{4}\right)$. Using now the expression of the system (3.14), Theorem 2.1, and the expressions (2.3) we can easily finish the proof of this point.

Consequently as said before (3.32) finishes the proof.

\section{Analysis of the GibBs phenomenon for nONLinear CUBiC SPlines}

In this section we will study the Gibbs phenomenon of nonlinear cubic spline interpolation given by (3.15) for the step function $f(t)$ in (4.1),

$$
f(t)= \begin{cases}0, & -1 \leq t<0 \\ a, & 0 \leq t<1\end{cases}
$$

with $a \in \mathbb{R}$, when uniform grid-spacing is used. Let's set $m=2 n$ in (3.15), then $h=1 / n$ if we consider that $t_{0}=-1, t_{n}=0$ and $t_{2 n}=1$. The values $D_{k}$ are symmetric with respect to $D_{n}$, as the step function that we are considering is symmetric. Thus, we only need to consider half of them. Then, using the natural boundary conditions, the system for the $D_{k}$ in (3) takes the form,

$$
\begin{aligned}
2 D_{0}+D_{1} & =0, \\
D_{k-1}+4 D_{k}+D_{k+1} & =0, \quad k=1, \ldots, n-2 \\
D_{n-2}+4 D_{n-1}+D_{n} & =0, \\
D_{n-1}+4 D_{n}+D_{n+1} & =0, \quad\left(D_{n-1}=D_{n}\right) .
\end{aligned}
$$

Let's consider the elimination of the Gibbs phenomenon for jump discontinuities. First of all let's remember the definition of the Gibbs phenomenon introduced by Gottlieb and Shu [25]. Given a punctually discontinuous function $f$ and its sampling $f^{h}$ defined by $f_{n}^{h}=f(n h)$, the Gibbs phenomenon deals with the convergence of the function $g$ based on $f^{h}$ towards $f$ when $h$ goes to 0 . It can be characterized by two features [25]:

(1) Away from the discontinuity the convergence is rather slow and for any point $x$,

$$
|f(x)-g(x)|=O(h) .
$$

(2) There is an overshoot, close to the discontinuity, that does not diminish with the reduction of $h$. Thus,

$$
\max |f(x)-g(x)| \text { does not tend to zero with } h .
$$


Taking into account Theorem 2.1 and the definition of the Gibbs phenomenon it is easy to prove the following theorem,

Theorem 4.1. The nonlinear spline obtained through (3.15) does not present Gibbs oscillations.

Proof. Let's analyze the right hand side of the system in (3.15). If we remember now that $\delta_{i}$ and $\delta_{i-1}$ are divided differences, we know that they are $O(1 / h)$ in the presence of a jump discontinuity. Taking into account property 5 of Proposition 3.8, we know that the right hand side of (3.15) will be,

$$
\|\mathbf{f}\|_{\infty}=O(1) .
$$

Then, the vector of derivatives $\mathbf{D}$ that results from solving (3.15) will be,

$$
\|\mathbf{D}\|_{\infty}=\left\|A^{-1} \mathbf{f}\right\|_{\infty}=O(1) .
$$

Now, looking at the expressions in (2.3) we obtain that,

$$
\begin{aligned}
d_{i} & =y_{i}, \\
c_{i} & =D_{i}=O(1), \\
a_{i} & =\frac{h D_{i+1}+D_{i} h+2 y_{i}-2 y_{i+1}}{h^{3}}=\frac{O(h)+O(h)+2 y_{i}-2 y_{i+1}}{h^{3}}, \\
b_{i} & =-\frac{h D_{i+1}+2 D_{i} h+3 y_{i}-3 y_{i+1}}{h^{2}}=-\frac{O(h)+2 O(h)+3 y_{i}-3 y_{i+1}}{h^{2}} .
\end{aligned}
$$

At smooth zones $y_{i+1}-y_{i}=O(h)$ so,

$$
\begin{aligned}
d_{i} & =y_{i} \\
c_{i} & =D_{i}=O(1), \\
b_{i} & =-\frac{h D_{i+1}+2 D_{i} h+3 y_{i}-3 y_{i+1}}{h^{2}}=-\frac{O(h)+2 O(h)-3 O(h)}{h^{2}}=\frac{O(h)}{h^{2}} \\
& =O\left(\frac{1}{h}\right), \\
a_{i} & =\frac{h D_{i+1}+D_{i} h+2 y_{i}-2 y_{i+1}}{h^{3}}=\frac{O(h)+O(h)-2 O(h)}{h^{3}}=\frac{O(h)}{h^{3}}=O\left(\frac{1}{h^{2}}\right),
\end{aligned}
$$

and if $x \in\left[x_{i}, x_{i+1}\right]$, the equation of the spline (2.1) transforms into,

$$
\begin{aligned}
g_{i}(x)= & a_{i}\left(x-x_{i}\right)^{3}+b_{i}\left(x-x_{i}\right)^{2}+c_{i}\left(x-x_{i}\right)+d_{i}=O\left(\frac{1}{h^{2}}\right) O\left(h^{3}\right) \\
& +O\left(\frac{1}{h}\right) O\left(h^{2}\right)+O(h)+y_{i}=y_{i}+O(h) .
\end{aligned}
$$

If there is a discontinuity at the interval $\left[x_{i}, x_{i+1}\right]$ then $y_{i+1}-y_{i}=O(1)$ and,

$$
\begin{aligned}
d_{i} & =y_{i}, \\
c_{i} & =D_{i}=O(1), \\
b_{i} & =-\frac{h D_{i+1}+2 D_{i} h+3 y_{i}-3 y_{i+1}}{h^{2}}=-\frac{O(h)+2 O(h)-3 O(1)}{h^{2}}=\frac{O(1)}{h^{2}} \\
& =O\left(\frac{1}{h^{2}}\right), \\
a_{i} & =\frac{h D_{i+1}+D_{i} h+2 y_{i}-2 y_{i+1}}{h^{3}}=\frac{O(h)+O(h)-2 O(1)}{h^{3}}=\frac{O(1)}{h^{3}}=O\left(\frac{1}{h^{3}}\right),
\end{aligned}
$$


and if $x \in\left[x_{i}, x_{i+1}\right]$, the equation of the spline (2.1) transforms into,

$$
\begin{aligned}
g_{i}(x)= & a_{i}\left(x-x_{i}\right)^{3}+b_{i}\left(x-x_{i}\right)^{2}+c_{i}\left(x-x_{i}\right)+d_{i}=O\left(\frac{1}{h^{3}}\right) O\left(h^{3}\right) \\
& +O\left(\frac{1}{h^{2}}\right) O\left(h^{2}\right)+O(h)+y_{i}=y_{i}+O(1) .
\end{aligned}
$$

This means that the perturbation introduced by the nonlinear spline is $O(h)$ except at the interval that contains the discontinuity.

Now we can try to prove that the nonlinear spline provides a prediction that is in the interval $\left[y_{i}, y_{i+1}\right]$ when $h$ goes to zero. In order to do so, we express the equation of the spline (2.1) as

$$
\begin{aligned}
g_{i}(x)= & a_{i}\left(x-x_{i}\right)^{3}+b_{i}\left(x-x_{i}\right)^{2}+c_{i}\left(x-x_{i}\right)+d_{i} \\
= & \frac{h D_{i+1}+D_{i} h+2 y_{i}-2 y_{i+1}}{h^{3}}\left(x-x_{i}\right)^{3}-\frac{h D_{i+1}+2 D_{i} h+3 y_{i}-3 y_{i+1}}{h^{2}}\left(x-x_{i}\right)^{2} \\
& +D_{i}\left(x-x_{i}\right)+y_{i} .
\end{aligned}
$$

Now we can do the change of variables used before, $s=\frac{x-x_{i}}{h}$, and we obtain

$$
\begin{aligned}
g_{i}(x) & =\left(h D_{i+1}+D_{i} h+2 y_{i}-2 y_{i+1}\right) s^{3}-\left(h D_{i+1}+2 D_{i} h+3 y_{i}-3 y_{i+1}\right) s^{2}+D_{i} h s+y_{i}, \\
& =\left(1-s^{2}(3-2 s)\right) y_{i}+s^{2}(3-2 s) y_{i+1}+\left(s^{3}-2 s^{2}+s\right) h D_{i}+\left(s^{3}-s^{2}\right) h D_{i+1} \\
& =b_{1}(s) y_{i+1}+b_{2}(s) y_{i}+b_{3}(s) h D_{i}+b_{4}(s) h D_{i+1},
\end{aligned}
$$

for $s \in[0,1]$. In this last expression it is easy to see that the first two terms can be interpreted as a weighted mean of $y_{i}$ and $y_{i+1}$ (as the element $b_{1}(s)=s^{2}(3-2 s)$ of the Hermite basis is always in the unit interval $b_{1}(s) \in[0,1]$ if $s \in[0,1]$, and $b_{2}(s)=1-b_{1}(s)$ ). Thus, the addition of the first two terms of the expression $(2.4)$ will take a value always in the interval $\left[y_{i}, y_{i+1}\right]$. The expression can also be reformulated as follows,

$$
g_{i}(x)=y_{i}+\left(s^{2}(3-2 s)\right)\left(y_{i+1}-y_{i}\right)+\left(s^{3}-2 s^{2}+s\right) h D_{i}+\left(s^{3}-s^{2}\right) h D_{i+1},
$$

where the first two terms of (4.13) amount to a dilation and a translation of the element $b_{1}(s)=s^{2}(3-2 s)$, that has a minimum at $s=0$ and a maximum at $s=1$, so it can not introduce Gibbs phenomenon, nor $b_{2}(s)$. Let's analyze the third and fourth terms of (4.13). We can see that $b_{3}(s)$ and $b_{4}(s)$ are oscillating functions so the apparition of Gibbs phenomenon can be explained due to the presence of large coefficients accompanying these two elements of the Hermite basis. In the case of the nonlinear spline in (3.15) we have already analyzed that $D_{i}=O(1)$. If this is the case, the two last terms in (4.13) go to zero when $h \rightarrow 0$. Thus, there can not be Gibbs oscillation.

Remark 4.2. The previous proof is perfectly valid for the translated mean in (3.18) if we take into account Proposition 3.14 and the definition of the $\epsilon$ given in (3.21). In the end, the proof presented is based on property 5 of Proposition 3.8 that, in practice, is equivalent to expression (3.19) for the translated mean.

The nonlinear spline does not present Gibbs oscillations for piecewise constant functions and the analysis of this case is particularly simple and intuitive. The system in (4.2)-(4.5) is homogeneous, so $D_{i}=0 \forall i$ is the trivial solution of the system. Thus, looking at the expressions in (2.3) we obtain that

$$
\begin{aligned}
d_{i} & =y_{i}, \\
c_{i} & =D_{i}=0, \\
a_{i} & =\frac{h_{i+1} D_{i+1}+D_{i} h_{i+1}+2 y_{i}-2 y_{i+1}}{h_{i+1}^{3}}=\frac{2 y_{i}-2 y_{i+1}}{h_{i+1}^{3}}, \\
b_{i} & =-\frac{h_{i+1} D_{i+1}+2 D_{i} h_{i+1}+3 y_{i}-3 y_{i+1}}{h_{i+1}^{2}}=-\frac{3 y_{i}-3 y_{i+1}}{h_{i+1}^{2}},
\end{aligned}
$$


and the equation of the spline (2.1) transforms into,

$$
\begin{aligned}
g_{i}(x)= & a_{i}\left(x-x_{i}\right)^{3}+b_{i}\left(x-x_{i}\right)^{2}+c_{i}\left(x-x_{i}\right)+d_{i}=\frac{2 y_{i}-2 y_{i+1}}{h_{i+1}^{3}}\left(x-x_{i}\right)^{3} \\
& -\frac{3 y_{i}-3 y_{i+1}}{h_{i+1}^{2}}\left(x-x_{i}\right)^{2}+y_{i} .
\end{aligned}
$$

The equation of the spline is

$$
g_{i}(x)= \begin{cases}\frac{-2 a}{h_{i+1}^{3}}\left(x-x_{i}\right)^{3}+\frac{3 a}{h_{i+1}^{2}}\left(x-x_{i}\right)^{2}+y_{i}, & i=n-1, \\ y_{i}, & \text { other case }\end{cases}
$$

If we parametrize this expression, using the change of variables $s=\frac{x-x_{i}}{h_{i}}$, we obtain,

$$
g_{i}(s)= \begin{cases}a s^{2}(3-2 s)+y_{i}, & i=n-1, \\ y_{i}, & \text { other case }\end{cases}
$$

for $s \in[0,1]$. It is clear that the polynomial that we obtain for $i=n-1$ is a dilation and translation of the element $b_{1}(s)=s^{2}(3-2 s)$ of the Hermite basis. Therefore, it presents a minimum for $s=0$ and a maximum for $s=1$ in the interval $\left[x_{n-1}, x_{n}\right]$, so it does not oscillate. Thus, the resultant spline does not present Gibbs oscillations for piecewise continuous functions.

Remark 4.3. The previous proof is perfectly valid for the translated mean in (3.18). Taking again into account Proposition 3.14 and the definition of the $\epsilon$ given in (3.21), the only difference would be that $c_{i}=D_{i}=O\left(h^{2}\right)$ but the result of the proof would be the same.

\section{NUMERICAL EXPERIMENTS}

In this section we will present some numerical results that allow to conclude the validity of the theoretical results presented in the article. More specifically we will show that the nonlinear cubic splines eliminate the Gibbs phenomenon that appears when using cubic splines and that they keep the order of accuracy of cubic splines at smooth zones. As it has been shown in Section 4, the nonlinear spline does not present Gibbs oscillations. We will also test the performance of the new algorithm in the presence of noise. The functions used in this section have been plotted in Figure 1. At the boundaries we have chosen the natural boundary condition. Any other condition could have been chosen, as we are only interested in what happens close to the discontinuity.

\subsection{Uniform grid-spacing}

In this subsection we have chosen a uniform grid-spacing. The original functions have been sampled with 2048 points in the interval $[-1,1]$, thus the grid spacing is set to $h=\frac{2}{2047}$ for all the experiments. Then they have been subsampled taking one of every 16 samples (here we can choose any number of samples, as we have the expression of the spline and we can evaluate it at any point of the domain). This resolution has been used for all the experiments in this section. Then we reconstruct using the information of the subsampled functions and we compare with the data at the highest resolution. The original subsampled data has been represented with red filled circles, the original high resolution data with blue crosses and the reconstruction with black points. We are interested in determining if Gibbs phenomenon appears in the limit function obtained through linear and nonlinear cubic splines. Moreover we will measure the size of the overshoot introduced by cubic splines and compare it with the theoretical estimation obtained in Section B. As mentioned in Remark 3.17, all the numerical experiments presented for the uniform grid spacing have been done using the nonlinear translated mean (3.16) with the translation defined in (3.18) and the $\epsilon$ defined in (3.21). 

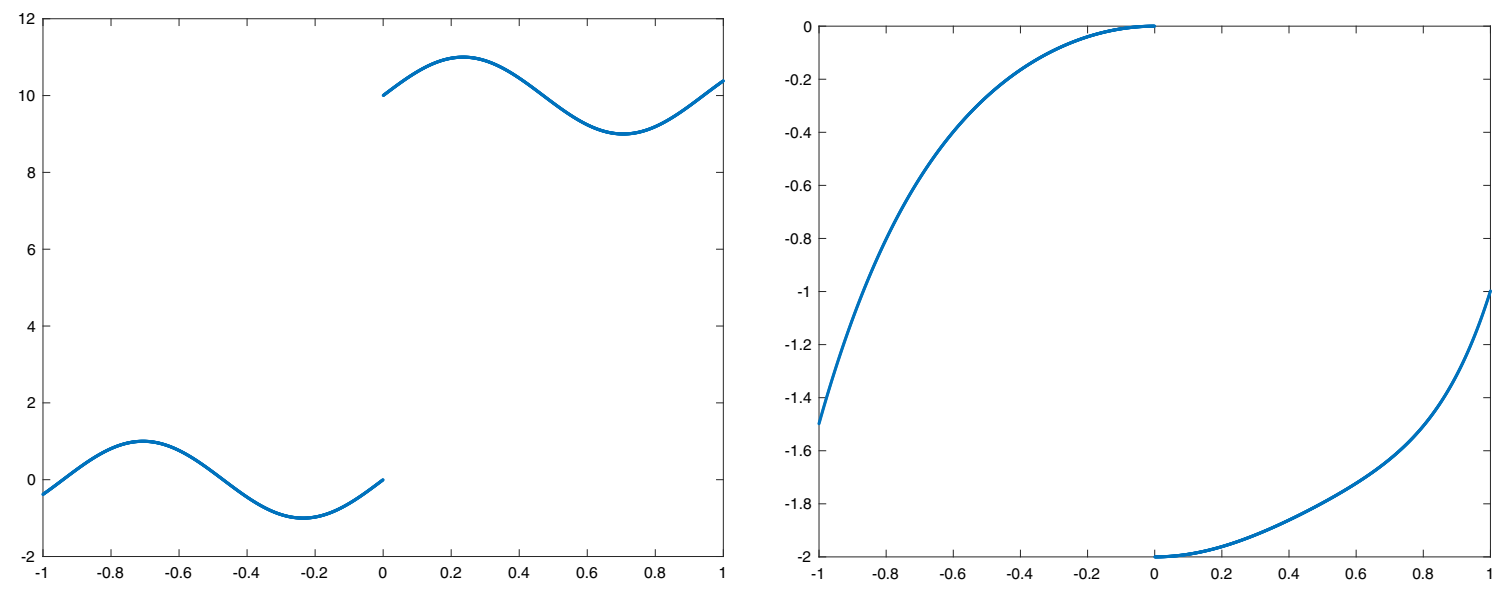

FiguRE 1. Functions in (5.1) and (5.2) used for the experiments presented in this section.

Example 5.1. Let's start by the function,

$$
f(x)= \begin{cases}\sin \left(\frac{17}{8} \pi x\right) & \text { if } x \leq 0, \\ \frac{1}{2} \sin \left(\frac{17}{8} \pi x\right)+10 & \text { if } x>0,\end{cases}
$$

presented in Figure 1, to the left. The results have been plotted in Figure 2, where at the top we can see the results for the nonlinear spline and at the bottom the results for the classical cubic spline. We can see that the oscillations that appear in the classical spline have been reduced in the results obtained by the nonlinear spline. In fact, these oscillations go to zero as the grid is refined. Thus, they do not satisfy the conditions described in [25] (and reproduced in Sect. 4) to be considered Gibbs phenomenon. For this example, the Gibbs oscillations that appear in the results obtained by the cubic spline present a maximum overshoot that is $10.8 \%$ of the jump in the function. This result agrees quite good with the bound given in (B.22). We observe that both methods introduce diffusion close to jump discontinuities in the function. In the case that the discontinuity in the original data is numerical, i.e. it is produced due to a not high enough sampling frequency close to high gradients in the function, it is good that the method introduces diffusion, as the diffusion is also present in the function from which the data was obtained.

Now we can check the performance of the linear and nonlinear cubic splines in the presence of noise. If we add white gaussian noise of zero mean and amplitude 0.1 to the function in (5.1), we obtain the results shown in Figure 3. We can appreciate that the Gibbs phenomenon is eliminated even in the presence of noise.

Example 5.2. Let's finish this section with the function,

$$
l(x)= \begin{cases}\frac{1}{2} x^{5}-x^{2} & \text { if } x \leq 0 \\ x^{6}-x^{4}+x^{2}-2 & \text { if } x>0 .\end{cases}
$$

The results are presented in Figure 4. We can observe that the maximum overshoot obtained by the cubic spline is the $10.78 \%$ of the jump, that again is inside the interval given in (B.22). In the results of both approaches we can observe diffusion, as the proposed technique is not designed to avoid it. The comments that we did about diffusion in the previous experiment can also be done here.

As before, we can check the performance of the cubic and nonlinear cubic splines in the presence of noise. If we add white gaussian noise of zero mean and amplitude 0.1 to the function in (5.2) we obtain the results shown in Figure 5. The results are similar as before and the Gibbs phenomenon is eliminated even in the presence of noise. 

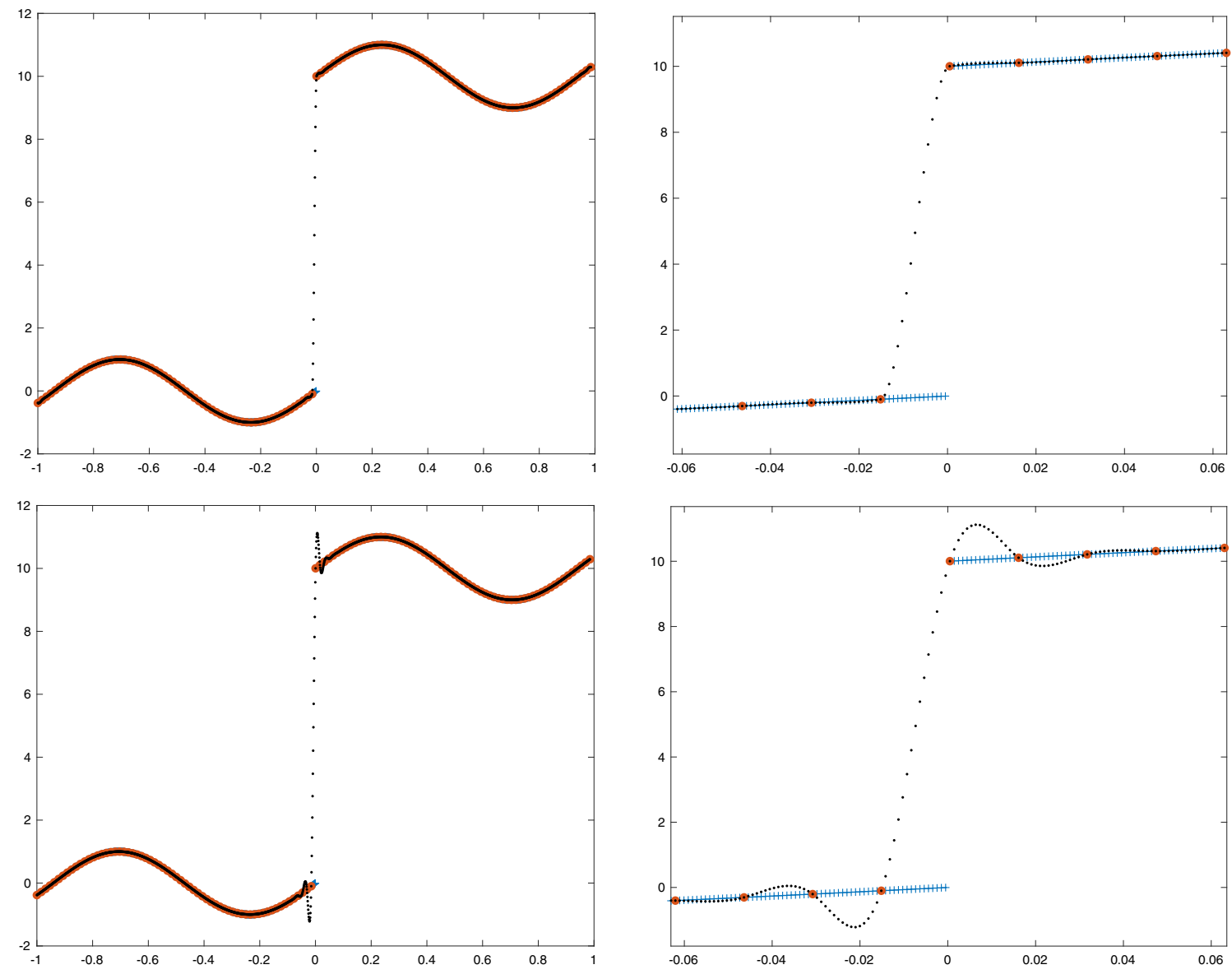

FiguRE 2. Top: Function in (5.1) reconstructed with an adapted cubic spline. Bottom: Same function reconstructed with a non-adapted cubic spline. In both cases, we have presented to the left the whole domain and to the right a zoom around the discontinuity. The low resolution discretization (red filled circles) has 128 points and the reconstruction (black points) 2048 points. The original high resolution data has been represented with blue crosses. We can clearly appreciate that Gibbs phenomenon appears in the result of the cubic spline. We also observe a very small oscillation in the reconstruction obtained through the nonlinear spline. It turns out that the oscillation is $O\left(h^{4}\right)$ and that goes to zero when $h$ goes to zero. (Color online).

\subsection{Grid refinement analysis}

In this subsection we present an experiment oriented to check the order of accuracy of the nonlinear cubic splines close to critical points at smooth zones of the function. In order to do this, we check the error of interpolation obtained at every grid point inside an interval around the discontinuity and then perform a grid refinement analysis. We define the order of accuracy of the reconstruction as,

$$
\operatorname{order}_{m}=\log _{2}\left(\frac{E_{m}}{E_{m+1}}\right)
$$

being $E_{m}$ the error obtained with a grid spacing $h_{m}$ and $E_{m+1}$ the error obtained with a grid spacing $h_{m} / 2$. 

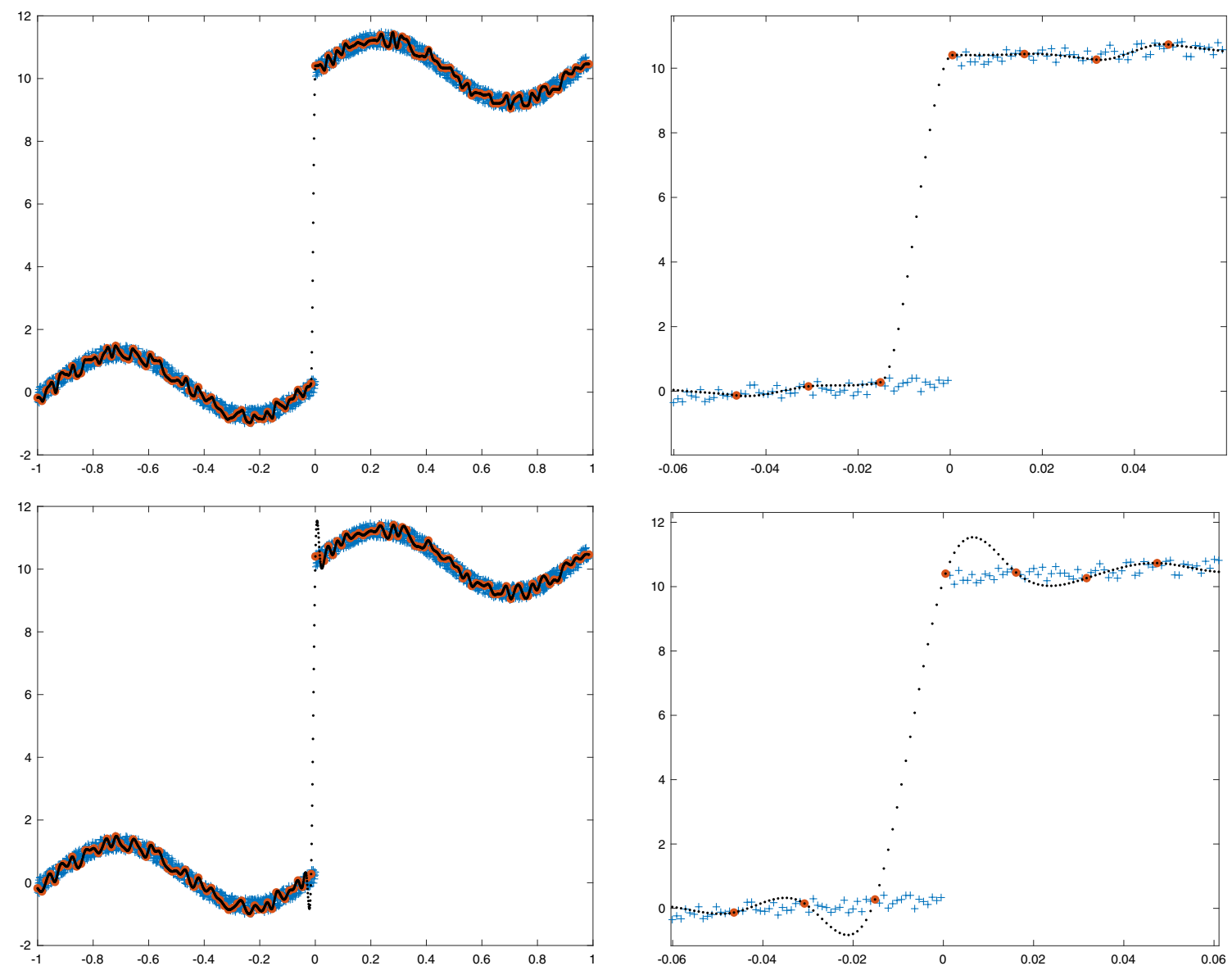

FiguRE 3. Top: Function in (5.1) plus additive white gaussian noise of amplitude 0.1 reconstructed with an adapted cubic spline. Bottom: Same function reconstructed with a non-adapted cubic spline. In both cases, we have presented to the left the whole domain and to the right a zoom around the discontinuity. The low resolution discretization (red filled circles) has 128 points and the reconstruction (black points) 2048 points. The original high resolution data has been represented with blue crosses. (Color online).

We will use the discrete $l_{\infty}$ norm,

$$
\|f\|_{\infty}=\max _{j \in \mathbb{Z}}\left\{\left|f_{j}\right|\right\} .
$$

Taking a fine mesh $\left\{x_{j}\right\}_{j \in H}$ of a small interval around the critical point not crossing any discontinuity, we compute

$$
E_{m}=\max _{j \in H}\left\|f_{j}-s_{j}\right\|_{\infty}
$$

where $f_{j}=f\left(x_{j}\right)$ and $s_{j}=s_{m}\left(x_{j}\right)$.

Example 5.3. Let's consider the function

$$
f(x)=\cos \left(\frac{3 \pi}{2} x\right),
$$



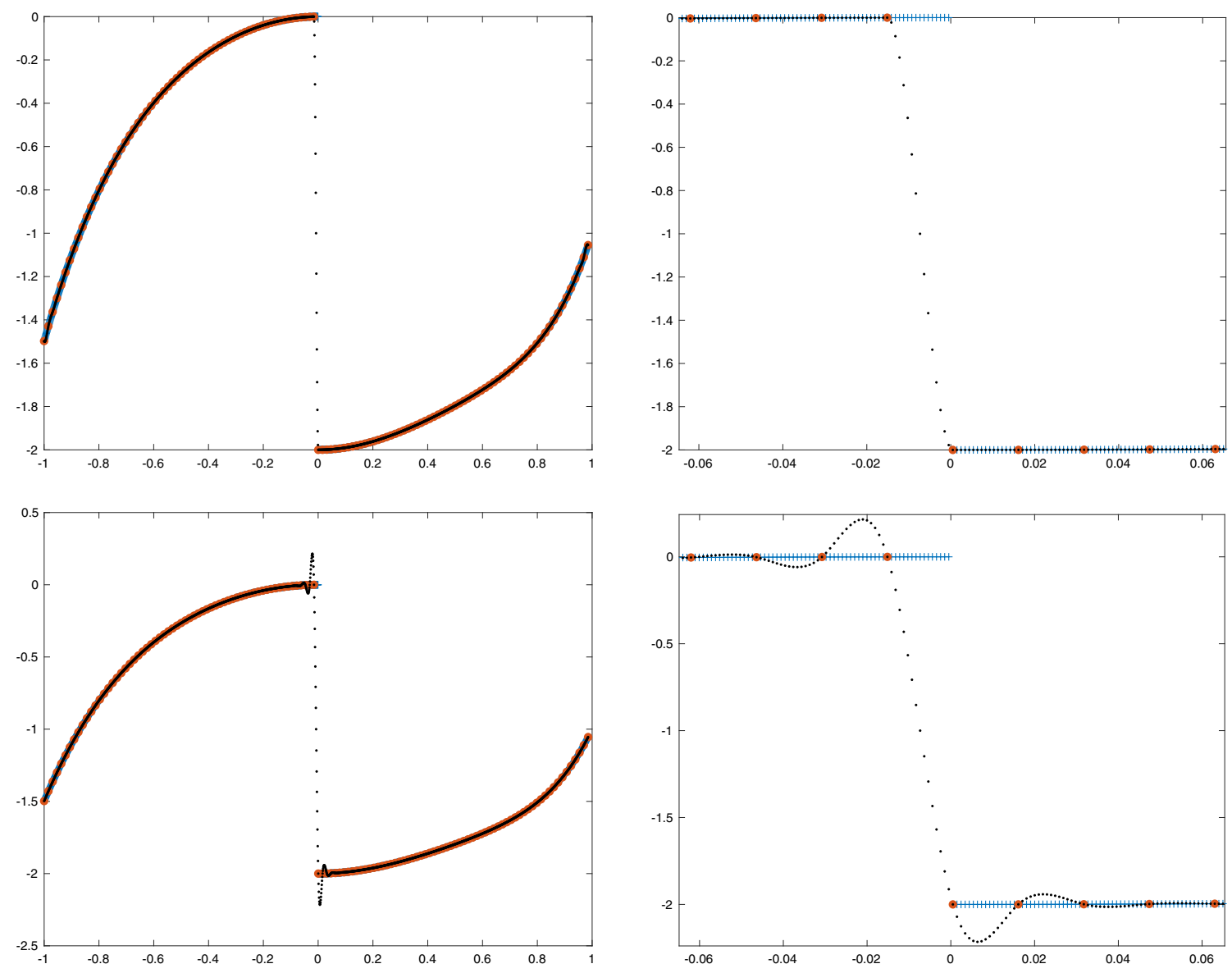

Figure 4. Top: Function in (5.2) reconstructed with an adapted cubic spline. Bottom: Same function reconstructed with a non-adapted cubic spline. In both cases, we have presented to the left the whole domain and to the right a zoom around the discontinuity. The low resolution discretization (red filled circles) has 128 points and the reconstruction (black points) 2048 points.The original high resolution data has been represented with blue crosses. (Color online).

that has been plotted in Figure 6 . We will check the accuracy of the nonlinear spline in the $l^{\infty}$ norm close to the critical point at $x=0$. The result is presented in Table 1 . We can see that the accuracy is order four, just as the theoretical order attained by the cubic splines at smooth zones.

Example 5.4. Let's consider again the function in (5.1). In this experiment we want to check if the order of accuracy at critical points placed at smooth zones are affected by the presence of discontinuities. A grid refinement analysis for the critical point placed at $x=-12 / 17 \approx-0.7$ is presented in Table 2 . We can see how the accuracy of the spline at smooth zones close to critical points is not affected by the discontinuity thanks to the translation proposed in Section 3.2.

\subsection{Non uniform grid-spacing}

In this subsection we have chosen a non-uniform grid-spacing. The original functions have been sampled with 2048 points in the interval $[-1,1]$ at random positions determined by a uniform distribution in that interval. 

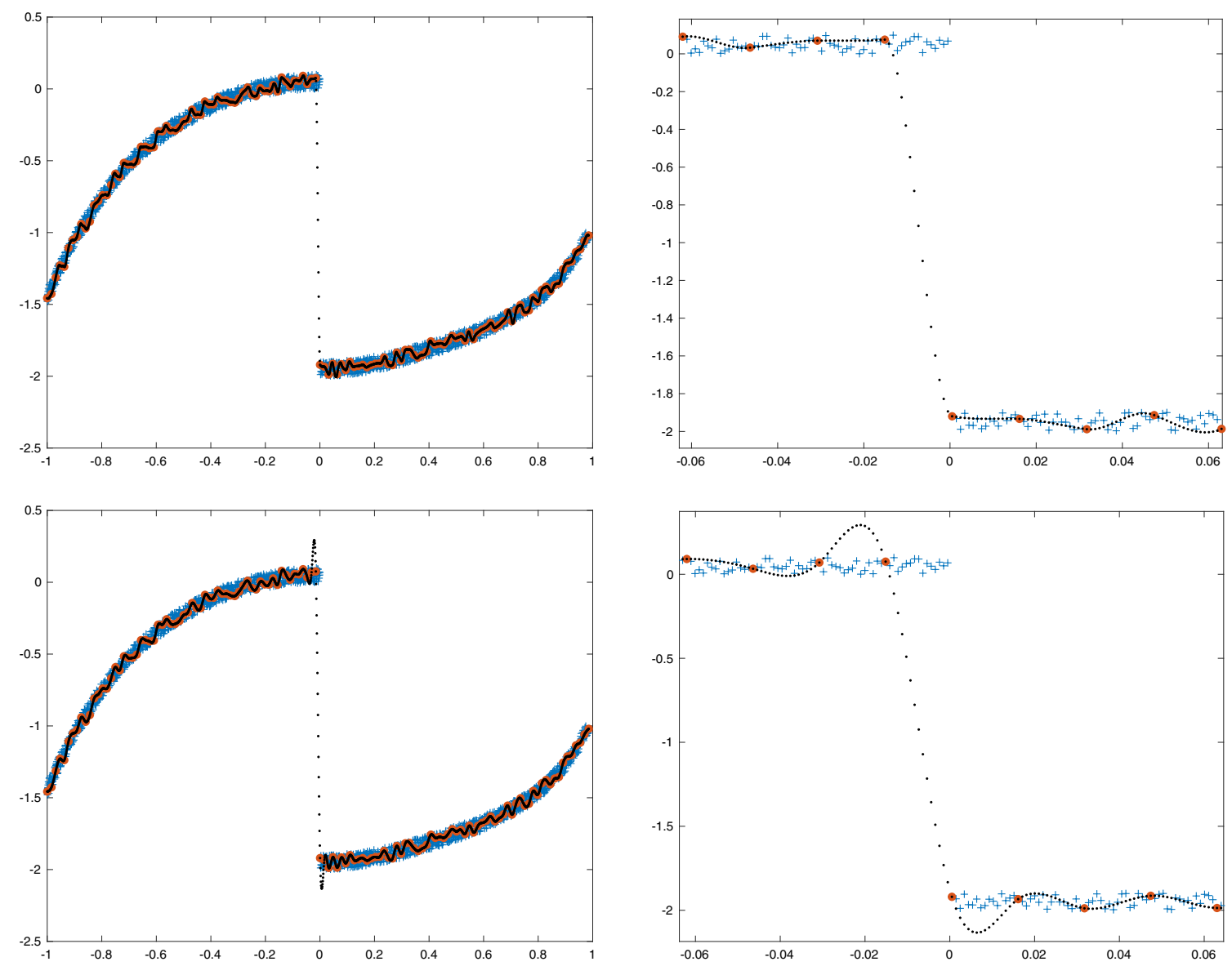

Figure 5. Top: Function in (5.2) plus additive white gaussian noise of amplitude 0.1 reconstructed with an adapted cubic spline. Bottom: Same function reconstructed with a non-adapted cubic spline. In both cases, we have presented to the left the whole domain and to the right a zoom around the discontinuity. The low resolution discretization (blue red circles) has 128 points and the reconstruction (black points) 2048 points. The original high resolution data has been represented with blue crosses. (Color online).

Then they have been subsampled to 128 points taking one of every 16 samples of the original discretization. The reconstruction is obtained keeping the sampling positions of the original discretization. The original subsampled data has been represented with red filled circles, the original high resolution data with blue crosses, and the reconstruction with black points. Now, we are only interested in the apparition of Gibbs phenomenon in the limit function. Thus, no translation have been used in the nonlinear mean.

Example 5.5. Let's consider again the function in (5.1). The results have been plotted in Figure 7, where at the top we can see the results for the nonlinear spline and at the bottom the results for the classical cubic spline. The conclusions that can be obtained from this experiment are the same that we obtained for the uniform grid spacing: the Gibbs phenomenon disappears from the result of the nonlinear spline. We can observe a very small oscillation that goes to zero when the mesh is refined. The overshoot obtained for the cubic spline is about $10.84 \%$ of the jump, that agrees with the bound obtained for the uniform case in (B.22), although that formula is only valid for the uniform case. 


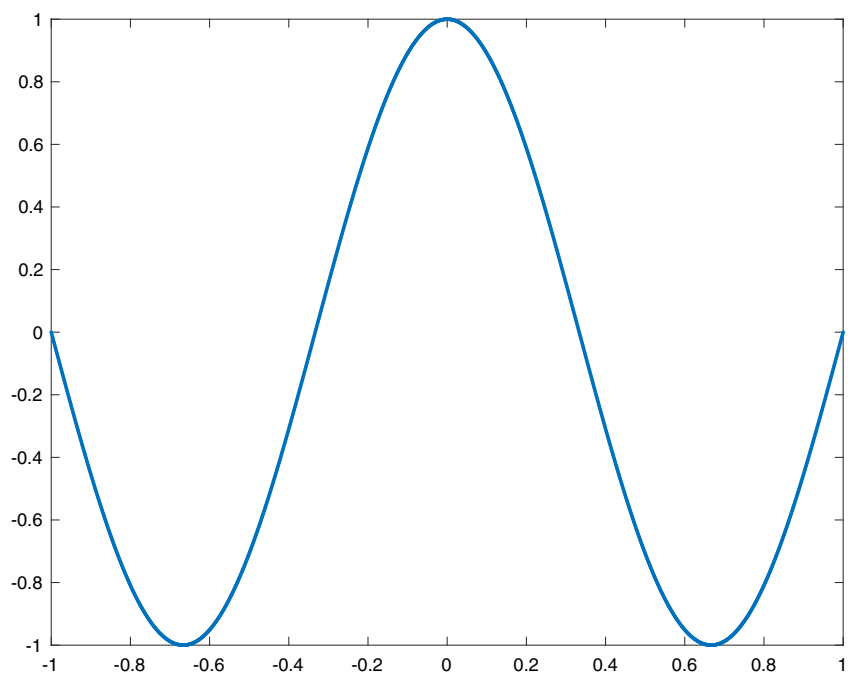

Figure 6. Function in (5.3), used for the grid refinement analysis presented in Table 1.

TABLE 1. Grid refinement analysis in the $l^{\infty}$ norm for the new nonlinear spline close to the critical point at $x=0$ of the function in (5.3).

\begin{tabular}{lll}
\hline \hline$m$ & $E_{m}$ & Order $_{m}$ \\
\hline 8 & $1.8025 \mathrm{e}-1$ & - \\
16 & $4.8849 \mathrm{e}-2$ & 1.8836 \\
32 & $5.1682 \mathrm{e}-3$ & 3.2406 \\
64 & $3.8821 \mathrm{e}-4$ & 3.7348 \\
128 & $2.9223 \mathrm{e}-5$ & 3.7317 \\
256 & $2.0865 \mathrm{e}-6$ & 3.8080 \\
512 & $1.7392 \mathrm{e}-7$ & 3.5845 \\
1024 & $1.0382 \mathrm{e}-8$ & 4.0664 \\
\hline
\end{tabular}

TABLE 2. Grid refinement analysis in the $l^{\infty}$ norm for the new nonlinear spline close to the critical point at $x=-12 / 17 \approx-0.7$ of the function in (5.1).

\begin{tabular}{lll}
\hline \hline$m$ & $E_{m}$ & Order $_{m}$ \\
\hline 8 & $3.6224 \mathrm{e}-1$ & - \\
16 & $2.4647 \mathrm{e}-1$ & 0.55551 \\
32 & $7.3385 \mathrm{e}-2$ & 1.7479 \\
64 & $1.5542 \mathrm{e}-2$ & 2.2393 \\
128 & $1.0265 \mathrm{e}-3$ & 3.9203 \\
256 & $4.114 \mathrm{e}-05$ & 4.6411 \\
512 & $4.8575 \mathrm{e}-06$ & 3.0823 \\
1024 & $2.4875 \mathrm{e}-07$ & 4.2874 \\
\hline
\end{tabular}



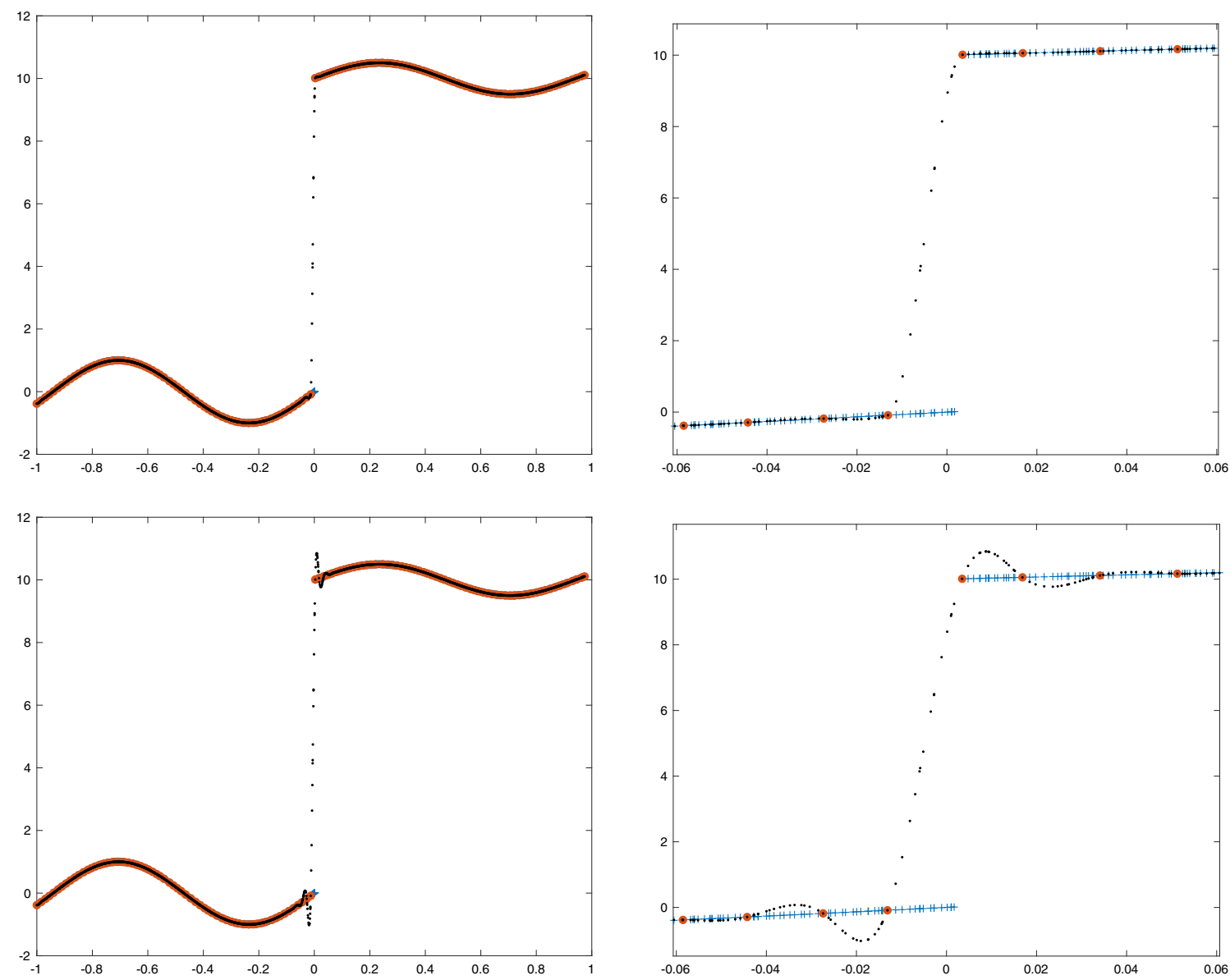

FiguRE 7. Top: Function in (5.1) reconstructed with an adapted cubic spline in a non-uniform grid. The discretization has been done using random positions determined by a uniform distribution in the interval $[-1,1]$. Bottom: Same function reconstructed with a non-adapted cubic spline. In both cases, we have presented to the left the whole domain and to the right a zoom around the discontinuity. The low resolution discretization (red filled circles) has 128 points and the reconstruction (black points) 2048 points. The original high resolution data has been represented with blue crosses. (Color online).

Example 5.6. Let's finish this subsection considering again the function in (5.2). The results have been plotted in Figure 8, where at the top we can see the results for the nonlinear spline and at the bottom the results for the classical cubic spline. These results are qualitatively the same as those obtained in previous subsection. Gibbs phenomenon does not appear in the reconstruction obtained using the nonlinear spline. The overshoot obtained for the cubic spline is around the $8.92 \%$ of the jump. In this case, the percentage is also inside the limits provided for the uniform grid-spacing.

\section{Conclusions}

We have presented a new class of nonlinear cubic splines that are automatically adapted to the presence of jump discontinuities in the function. The adaption is attained through the introduction of a limiter in the right 

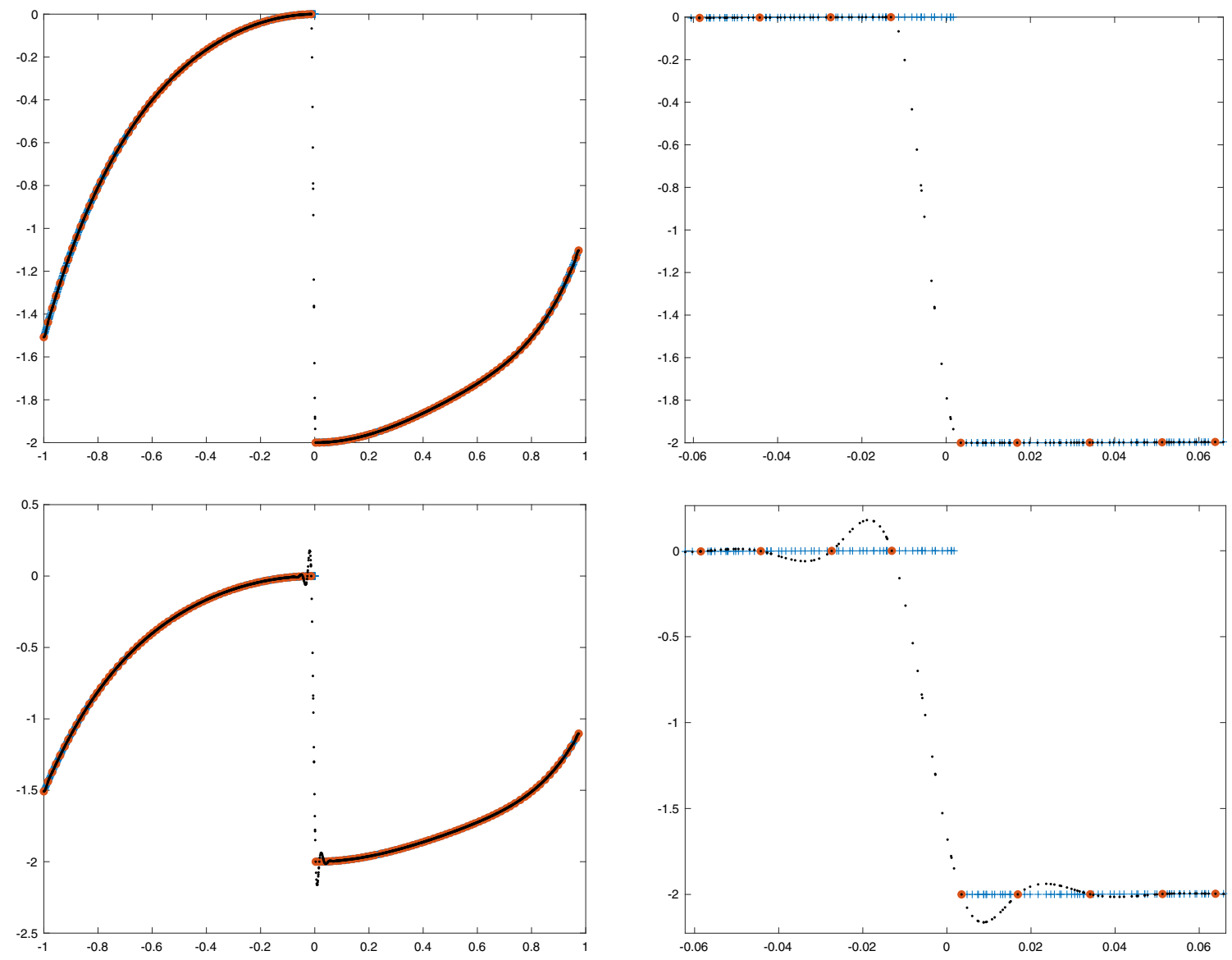

FiguRE 8. Top: Function in (5.1) reconstructed with an adapted cubic spline in a non-uniform grid. The discretization has been done using random positions determined by a uniform distribution in the interval $[-1,1]$. Bottom: Same function reconstructed with a non-adapted cubic spline. In both cases, we have presented to the left the whole domain and to the right a zoom around the discontinuity. The low resolution discretization (red filled circles) has 128 points and the reconstruction (black points) 2048 points. The original high resolution data has been represented with blue crosses. (Color online).

hand side of the system of equations of the spline. As far as we know, it is the first time that this limiter appears in the literature and that this technique is used to adapt splines to the presence of discontinuities. We have analyzed theoretically the properties of the limiter concluding that it can be used to attain adaption close to discontinuities. We have proved theoretically that the new class of splines presented is free of Gibbs oscillations and that it maintains the diffusion in the interval that contains the discontinuity. The diffusion introduced is an advantage when the discontinuity is numerical, that means that it is produced in the discretization process, due to a not high enough sampling frequency close to high gradients in the function. In the computer aided design (CAD) field, this kind of discontinuities are usually solved through a post-processing using a local refinement of the grid once the result is obtained and the numerical artifacts are observed. The new technique allows to solve these problems automatically.

We have also proved that cubic splines loss their accuracy close to discontinuities and we have given an explanation of why this phenomenon occurs. We have given a bound for the oscillations of the cubic splines 
close to the jump discontinuity of a step function in the uniform case. The given bound agrees very well with the performed numerical tests. In fact all the numerical experiments presented for the uniform case agree very well with the theoretical results analyzed. In the non-uniform case, the numerical experiments seem to satisfy bounds for the Gibbs oscillations that are similar to those obtained for the uniform case.

\section{Appendix A. Analysis of the GibBs phenomenon for Cubic splines}

In this section we follow the proof in [39] where the authors prove that cubic splines do not present Gibbs oscillations when interpolating a step function with a value in the middle of the jump. Our case is different, as we are interested in analyzing piecewise smooth functions.

Theorem A.1. The cubic spline presents Gibbs oscillations for piecewise smooth functions with jump discontinuities.

Proof. We can follow the proof presented before for nonlinear cubic splines. Let's analyze the right hand side of the system in (3.14). If we remember now that $\delta_{i}$ and $\delta_{i-1}$ are divided differences, we know that they are $O(1 / h)$ in the presence of a jump discontinuity. The right hand side of (3.14) will be,

$$
\|\mathbf{f}\|_{\infty}=O\left(\frac{1}{h}\right) .
$$

Then, the vector of derivatives $\mathbf{D}$ that results from solving (3.14) will be,

$$
\|\mathbf{D}\|_{\infty}=\left\|A^{-1} \mathbf{f}\right\|_{\infty} \leq\left\|A^{-1}\right\|_{\infty}\|\mathbf{f}\|_{\infty} \leq O(1) O\left(\frac{1}{h}\right)=O\left(\frac{1}{h}\right)
$$

since $A$ is a symmetric strictly diagonal dominant matrix. Now, looking at the expressions of the coefficients of the spline in (2.3) we obtain that,

$$
\begin{aligned}
& d_{i}=y_{i}, \\
& c_{i}=D_{i}=O\left(\frac{1}{h}\right), \\
& a_{i}=\frac{h D_{i+1}+D_{i} h+2 y_{i}-2 y_{i+1}}{h^{3}}=\frac{O(1)+O(1)+2 y_{i}-2 y_{i+1}}{h^{3}}, \\
& b_{i}=-\frac{h D_{i+1}+2 D_{i} h+3 y_{i}-3 y_{i+1}}{h^{2}}=-\frac{O(1)+2 O(1)+3 y_{i}-3 y_{i+1}}{h^{2}} .
\end{aligned}
$$

At smooth zones $y_{i+1}-y_{i}=O(h)$ so,

$$
\begin{aligned}
& d_{i}=y_{i}, \\
& c_{i}=D_{i}=O\left(\frac{1}{h}\right) \\
& b_{i}=-\frac{h D_{i+1}+2 D_{i} h+3 y_{i}-3 y_{i+1}}{h^{2}}=-\frac{O(1)+2 O(1)-3 O(h)}{h^{2}}=O\left(\frac{1}{h^{2}}\right), \\
& a_{i}=\frac{h D_{i+1}+D_{i} h+2 y_{i}-2 y_{i+1}}{h^{3}}=\frac{O(1)+O(1)-2 O(h)}{h^{3}}=O\left(\frac{1}{h^{3}}\right),
\end{aligned}
$$

and if $x \in\left[x_{i}, x_{i+1}\right]$, the equation of the spline (2.1) transforms into,

$$
g_{i}(x)=a_{i}\left(x-x_{i}\right)^{3}+b_{i}\left(x-x_{i}\right)^{2}+c_{i}\left(x-x_{i}\right)+d_{i}=y_{i}+O(1),
$$

since $\left(x-x_{i}\right)=O(h)$ if $x \in\left[x_{i}, x_{i+1}\right]$. 
If there is a discontinuity in the interval $\left[x_{i}, x_{i+1}\right]$ then $y_{i+1}-y_{i}=O(1)$ so,

$$
\begin{aligned}
& d_{i}=y_{i} \\
& c_{i}=D_{i}=O\left(\frac{1}{h}\right) \\
& b_{i}=-\frac{h D_{i+1}+2 D_{i} h+3 y_{i}-3 y_{i+1}}{h^{2}}=-\frac{O(1)+2 O(1)-3 O(1)}{h^{2}}=O\left(\frac{1}{h^{2}}\right), \\
& a_{i}=\frac{h D_{i+1}+D_{i} h+2 y_{i}-2 y_{i+1}}{h^{3}}=\frac{O(1)+O(1)-2 O(1)}{h^{3}}=O\left(\frac{1}{h^{3}}\right)
\end{aligned}
$$

and if $x \in\left[x_{i}, x_{i+1}\right]$, the equation of the spline (2.1) transforms into,

$$
g_{i}(x)=a_{i}\left(x-x_{i}\right)^{3}+b_{i}\left(x-x_{i}\right)^{2}+c_{i}\left(x-x_{i}\right)+d_{i}=y_{i}+O(1) .
$$

It is easy to make an interpretation of this result: equation (2.4) is valid for the cubic spline and, after (A.1), we can see that in this case $D_{i}=D_{i+1}=O\left(\frac{1}{h}\right)$ in the $l_{\infty}$ norm, so the oscillations introduced by the two Hermite base functions $b_{3}(s)=s^{3}-2 s^{2}+s$ and $b_{4}(s)=s^{3}-s^{2}$ do not go away when $h$ goes to zero. Using the definition introduced by Gottlieb and Shu in [25], we can conclude that the precision of the spline is lost due to the apparition of Gibbs oscillations.

\section{Appendix B. Estimation of the overshoot of the GibBs phenomenon for Cubic SPLINES}

In this section we will try to estimate the overshoot that cubic splines introduce close to jump discontinuities in the function. In order to do so, we follow the proof introduced in [39]. It is important to remark that the bounds given for the oscillations in [39] are not valid in our case since the authors use for the proofs a step function that has a value in the middle of the jump discontinuity.

\section{B.1. $L^{p}$ convergence}

We want to interpolate the step function,

$$
f(t)= \begin{cases}0, & -1 \leq t<0 \\ a, & 0 \leq t<1\end{cases}
$$

with $a>0$ without loss of generality.

In [39], the authors prove Theorem 2.1 and the following theorem,

Theorem B.1. If we denote by $h=\max _{1 \leq i \leq n} h_{i}$, then $\left\|s_{n}-f\right\|_{p} \rightarrow 0$ with rate $h^{1 / p}$ if

$$
\left(\sum_{k=1}^{n}\left|h_{k} D_{k-1}\right|^{p}+\left|h_{k} D_{k}\right|^{p}\right)^{1 / p}
$$

is bounded uniformly with respect to $n, 1 \leq p<\infty$, where $s_{n}(x)=g_{i}(x)$ in (2.1) when $x \in\left[x_{i}, x_{i+1}\right]$.

A sequence of meshes is called quasi-uniform if there exist $\sigma>0$ independent of $n$, such that $\frac{\max _{i} h_{i}}{\min _{j} h_{j}} \leq \sigma$.

Theorem B.2. If we assume that the mesh is quasi-uniform, then $\left\|s_{n}-f\right\|_{p} \rightarrow 0$ with rate $h^{1 / p}$ for $1 \leq p<\infty$. 
Proof. A sufficient condition for Theorem B.1 to hold is that $h\|\mathbf{D}\|_{p} \leq C$, where $C$ is a constant independent of $h$. Then, we need to estimate

$$
\|\mathbf{D}\|_{p} \leq\left\|A^{-1}\right\|_{p}\|\mathbf{f}\|_{p} .
$$

We already have an upper bound for $\left\|A^{-1}\right\|_{p}$ using Theorem 2.1. From (2.7) and using the step function shown in (4.1), it is straightforward to verify that the right hand side for this particular case is,

$$
\mathbf{f}=\left(0, \ldots, 0, \frac{3 a}{h_{n}^{2}}, \frac{3 a}{h_{n}^{2}}, 0, \ldots, 0\right)^{T} .
$$

Then, we can obtain the expression for $\|\mathbf{f}\|_{p}$,

$$
\|\mathbf{f}\|_{p}=\frac{3 a}{h_{n}^{2}} 2^{\frac{1}{p}}
$$

Then, using Theorem 2.1, we obtain that,

$$
\|\mathbf{D}\|_{p} \leq \max _{i}\left(\frac{1}{h_{i}}+\frac{1}{h_{i+1}}\right)^{-1}\|\mathbf{f}\|_{p},
$$

and we finish the proof.

\section{B.2. A bound for the overshoot of cubic splines close to a jump discontinuity}

In this section we will study the Gibbs phenomenon of cubic spline interpolation for the step function $f$ in (4.1) when a uniform grid-spacing is used. Let's set $m=2 n$ in (2.7), then $h=1 / n$ if we consider that $t_{0}=-1, t_{n}=0$ and $t_{2 n}=1$, having in mind that we are going to interpolate the function in (4.1). The values $D_{k}$ are symmetric with respect to $D_{n}$, as the step function that we are considering is symmetric. Thus, we only need to consider half of them. Then, the system for the $D_{k}$ in (3) takes the form,

$$
\begin{aligned}
2 D_{0}+D_{1} & =0, \\
D_{k-1}+4 D_{k}+D_{k+1} & =0, \quad k=1, \ldots, n-2 \\
D_{n-2}+4 D_{n-1}+D_{n} & =\frac{3 a}{h}, \\
D_{n-1}+4 D_{n}+D_{n+1} & =\frac{3 a}{h}, \quad\left(D_{n-1}=D_{n}\right) .
\end{aligned}
$$

Before proving the main theorems of this section we need the following technical lemma.

Lemma B.3. Let us consider the sequence $\left\{c_{k}\right\}_{k \in \mathbb{N}}$ generated by

$$
\left\{\begin{array}{l}
c_{1} \in \mathbb{R} \text { given, } \\
c_{k+1}=\frac{1}{b-c_{k}},
\end{array}\right.
$$

where $b$ is any positive real number. The following statements are true,

(1) The iteration function $g(c)=\frac{1}{b-c}$ has two fixed points given by $c_{1}^{*}=\frac{b-\sqrt{b^{2}-4}}{2}$ and $c_{2}^{*}=\frac{b+\sqrt{b^{2}-4}}{2}$.

(2) If $c_{1}^{*}<c_{1}<c_{2}^{*}$ then the sequence is monotonically decreasing and converges to $c_{1}^{*}$.

(3) If $c_{1}<c_{1}^{*}$ then the sequence is monotonically increasing and converges to $c_{1}^{*}$.

Proof. Solving the equation $c=g(c)$ directly gives the two fixed points $c_{1}^{*}$ and $c_{2}^{*}$, and this proves the first statement.

We observe that $c_{k}^{2}-b c_{k}+1=\left(c_{k}-c_{1}^{*}\right)\left(c_{k}-c_{2}^{*}\right)$. Then if $c_{1}^{*}<c_{1}<c_{2}^{*}$, we have that $\frac{1}{b-c_{k}}<c_{k}$ and $\frac{1}{b-c y_{k}}>c_{1}^{*}$ for all $k \in \mathbb{N}$. Therefore the sequence is monotonically decreasing and has a lower bound, which implies using (B.9) that it converges to $c_{1}^{*}$, and we have proven the second statement. 
The proof for the third statement is similar. Since we start this time with $c_{1}<c_{1}^{*}$, we have that $\frac{1}{b-c_{k}}>c_{k}$ and $\frac{1}{b-c y_{k}}<c_{1}^{*}$ for all $k \in \mathbb{N}$. Thus the sequence is monotonically increasing and has an upper bound, which implies using (B.9) that it converges to $c_{1}^{*}$.

Theorem B.4. The $D_{k}$ 's present the following properties,

(1) They have alternating sign:

$$
D_{n-k}(-1)^{k}<0, \quad k=1, \ldots, n-1 .
$$

(2) Exponential decay:

$$
\frac{1}{4}\left|D_{k+1}\right|<\left|D_{k}\right|<\frac{2}{7}\left|D_{k+1}\right|, \quad k=1, \ldots, n-2 .
$$

(3) Upper and lower bounds:

$$
\begin{gathered}
\frac{45}{71} a<D_{n-1} h<\frac{26 a}{41}, \\
-\frac{7 a}{41}<D_{n-2} h<-\frac{12 a}{71} .
\end{gathered}
$$

(4) Asymptotical behavior:

$$
\begin{aligned}
\lim _{n \rightarrow \infty} h D_{n-2} & =-\frac{3 a}{19}, \\
\lim _{n \rightarrow \infty} h D_{n-1} & =\frac{12 a}{19}, \\
\lim _{n \rightarrow \infty} h D_{n-3} & =0 .
\end{aligned}
$$

Proof. Let's first show that $D_{0} \neq 0$. If we suppose that $D_{0}=0$, then $D_{1}=0$ from (B.5), and $D_{2}=0, \ldots, D_{n-1}=$ 0 from (B.6). Then, using (B.7) $D_{n-1}=\frac{3 a}{5 h}$. This a contradiction and $D_{0} \neq 0$.

If $D_{0} \neq 0$, then $D_{0} D_{1}<0$ and $\left|D_{1}\right|=2\left|D_{0}\right|$ using (B.5). Then, from (B.6) it is easy to see that $D_{0}+4 D_{1}+D_{2}=$ 0 and then $D_{1} D_{2}<0$ and,

$$
\left|4 D_{1}\right|=\left|-D_{2}-D_{0}\right|=\left|D_{2}\right|+\left|D_{0}\right|=\left|D_{2}\right|+\frac{1}{2}\left|D_{1}\right| .
$$

Thus $\left(4-\frac{1}{2}\right)\left|D_{1}\right|=\left|D_{2}\right|$, and therefore $\left|D_{1}\right|=\frac{2}{7}\left|D_{2}\right|$. Following the same argument we obtain recursively $\left|D_{k}\right|=c_{k+1}\left|D_{k+1}\right|$, with $c_{k}$ satisfying,

$$
\left\{\begin{array}{l}
c_{1}=\frac{1}{2} \\
c_{k+1}=\frac{1}{4-c_{k}}
\end{array}\right.
$$

Using Lemma B.3, since $2-\sqrt{3}<\frac{1}{2}<2+\sqrt{3}$, the sequence $\left\{c_{k}\right\}_{k \in \mathbb{N}}$ is, monotonically decreasing and converges to $2-\sqrt{3}$. Now, we get that,

$$
\frac{1}{4}\left|D_{k+1}\right| \leq \frac{1}{4-c_{k}}\left|D_{k+1}\right|=\left|D_{k}\right| \leq \frac{2}{7}\left|D_{k+1}\right| .
$$

We have seen that $D_{1}$ has different sign than $D_{2}$. By induction we suppose that $D_{l}$ has different sign that $D_{l-1}$ for any $l=3,4, \ldots, n-2$. Since $\left|4 D_{l}\right|=\frac{4}{4-c_{l-1}}\left|D_{l-1}\right|>\left|D_{l-1}\right|$, we get from the equation $D_{l-1}+4 D_{l}+D_{l+1}=0$ that $D_{l}$ has also different sign than $D_{l+1}$, and we have proved properties 1 and 2 of the theorem.

Now we can obtain $D_{n-2}$ and $D_{n-1}$ solving (B.6) and (B.7),

$$
\begin{aligned}
& D_{n-2} h=-\frac{5 h D_{n-3}+3 a}{19}, \\
& D_{n-1} h=\frac{h D_{n-3}+12 a}{19} .
\end{aligned}
$$


Remind now that $D_{n-3} D_{n-2}<0$ and $\left|D_{n-2}\right| / 4<\left|D_{n-3}\right|<\frac{2}{7}\left|D_{n-2}\right|$. From (B.19) we can see that there is no other option than $D_{n-3}>0$ to satisfy the condition $D_{n-3} D_{n-2}<0$. Then $D_{n-2}<0$. We also have that from (B.19),

$$
\begin{aligned}
-\frac{3 a}{19 h} & =D_{n-2}+\frac{5}{19} D_{n-3} \\
& =D_{n-2}+\frac{5}{19}\left|D_{n-3}\right|\left\{\begin{array}{l}
<D_{n-2}+\frac{5}{19} \frac{2}{7}\left|D_{n-2}\right|=-\frac{123}{133}\left|D_{n-2}\right|, \\
>D_{n-2}+\frac{5}{19} \frac{\left|D_{n-2}\right|}{4}=-\frac{71}{76}\left|D_{n-2}\right| .
\end{array}\right.
\end{aligned}
$$

Operating from the first inequality we easily obtain that,

$$
h\left|D_{n-2}\right|<\frac{7 a}{41},
$$

and manipulating the second inequality we arrive to,

$$
h\left|D_{n-2}\right|>\frac{12 a}{71} .
$$

Consequently we obtain that,

$$
-\frac{7 a}{41}<h D_{n-2}<-\frac{12 a}{71} .
$$

Now, using the previous relation and (B.7) we obtain that,

$$
D_{n-1} h=\frac{3 a}{5}-\frac{D_{n-2} h}{5}=\left\{\begin{array}{l}
<\frac{3 a}{5}+\frac{7 a}{205}=\frac{26 a}{41}, \\
>\frac{3 a}{5}+\frac{1}{5} \frac{12 a}{71}=\frac{45}{71} a,
\end{array}\right.
$$

so $\frac{45}{71} a<D_{n-1} h<\frac{26 a}{41}$. Thus, we have proved property 3 .

Again from Lemma B.3 and having into account that $h=\frac{1}{n}$ it follows that $D_{n-3}^{*}=h D_{n-3}$ is a monotonically decreasing sequence with

$$
\lim _{n \rightarrow \infty} D_{n-3}^{*}=\lim _{n \rightarrow \infty} h D_{n-3}=0 .
$$

Then from (B.19) $D_{n-2}^{*}=h D_{n-2}$ is a monotonically increasing sequence and

$$
\lim _{n \rightarrow \infty} D_{n-2}^{*}=\lim _{n \rightarrow \infty} h D_{n-2}=-\frac{3 a}{19} .
$$

And from (B.20) $D_{n-1}^{*}=h D_{n-1}$ is a monotonically decreasing sequence and

$$
\lim _{n \rightarrow \infty} D_{n-1}^{*}=\lim _{n \rightarrow \infty} h D_{n-1}=\frac{12 a}{19},
$$

and we have proved property 4 .

Theorem B.5. The overshoot for the cubic spline $g_{i}(t)$ close to the jump discontinuity of a step function $f$ is bounded by,

$$
0.1068 a<\max _{t_{k-1} \leq t \leq t_{k}}\left|g_{k-1}(t)-f(t)\right|<0.1089 a .
$$

Proof. From the properties obtained for the $D_{i}$ we can try to compute the size of the overshoot. We have already seen that we can express the spline in terms of the Hermite basis. Let's consider the expression of the $l_{\infty}$ norm of the error at the region where $f(t)=0$ in (4.1). Using (4.13), the expression of the error is,

$$
\begin{aligned}
\max _{t_{k-1} \leq t \leq t_{k}}\left|s_{n}(t)-f(t)\right| & =\max _{t_{k-1} \leq t \leq t_{k}}\left|D_{k-1} b_{3}\left(\frac{t-t_{k}}{h}\right) h+D_{k} b_{4}\left(\frac{t-t_{k}}{h}\right) h\right| \\
& =\left|D_{k}\right| h \max _{0 \leq s \leq 1}\left(-\frac{D_{k-1}}{D_{k}} b_{3}(s)-b_{4}(s)\right),
\end{aligned}
$$


where we have made use of the fact that $D_{k} D_{k-1}<0$ and $b_{4}(s)<0 \forall s \in[0,1]$.

Now we can make use of the exponential decay property (B.11) in order to see that,

$$
\begin{gathered}
\frac{1}{4} \leq-\frac{D_{k}}{D_{k+1}} \leq \frac{2}{7}, \\
4^{-(n-1-k)} D_{n-1} \leq\left|D_{k}\right| \leq\left(\frac{2}{7}\right)^{n-1-k} D_{n-1} .
\end{gathered}
$$

Now let's use the bound in (B.24) in (B.23). Then we can write the error functions,

$$
\begin{aligned}
e(s) & =\frac{1}{4} b_{3}(s)-b_{4}(s), \\
E(s) & =\frac{2}{7} b_{3}(s)-b_{4}(s) .
\end{aligned}
$$

Then it is easy to see that

$$
\begin{aligned}
& \max _{0 \leq s \leq 1} e(s)=e\left(\frac{\sqrt{13}+2}{9}\right)=\frac{35}{486}+\frac{13}{486} \sqrt{13} \\
& \max _{0 \leq s \leq 1} E(s)=E\left(\frac{3+\sqrt{39}}{15}\right)=\frac{12}{175}+\frac{26 \sqrt{39}}{1575} .
\end{aligned}
$$

Then, for $k=1, \ldots, n-1$,

$$
\frac{35}{486}+\frac{13}{486} \sqrt{13}<-\frac{D_{k-1}}{D_{k}} b_{3}(s)-b_{4}(s)<\frac{12}{175}+\frac{26 \sqrt{39}}{1575} .
$$

Now, we have an estimate of $D_{k} h$ from (B.25) and (B.12),

$$
4^{-n+1+k} \frac{45}{71} a<\left|D_{k} h\right|<\left(\frac{2}{7}\right)^{n-1-k} \frac{26 a}{41} .
$$

Replacing (B.26) and (B.27) in (B.23), we have that for $k=1, \ldots, n-1$,

$$
\begin{aligned}
\max _{t_{k-1} \leq t \leq t_{k}}\left|s_{n}(t)-f(t)\right| & <\left(\frac{2}{7}\right)^{n-1-k} \frac{26 a}{41}\left(\frac{12}{175}+\frac{26 \sqrt{39}}{1575}\right) \\
& <\left(\frac{2}{7}\right)^{n-1-k} 0.1089 a \\
\max _{t_{k-1} \leq t \leq t_{k}}\left|s_{n}(t)-f(t)\right| & >4^{-(n-1-k)} \frac{45}{71} a\left(\frac{35}{486}+\frac{13}{486} \sqrt{13}\right) \\
& >4^{-(n-1-k)} 0.1068 a .
\end{aligned}
$$

Now setting $k=n-1$ in (B.28) and (B.29), we have that the overshoot for the cubic spline is bounded by,

$$
0.1068 a<\max _{t_{k-1} \leq t \leq t_{k}}\left|s_{n}(t)-f(t)\right|<0.1089 a .
$$

Acknowledgements. We would like to thank the anonymous referees for their valuable comments, which have helped to significantly improve this work. This work was funded by project 20928/PI/18 (Proyecto financiado por la Comunidad Autónoma de la Región de Murcia a través de la convocatoria de Ayudas a proyectos para el desarrollo de investigación científica y técnica por grupos competitivos, incluida en el Programa Regional de Fomento de la Investigación Científica y Técnica (Plan de Actuación 2018) de la Fundación Séneca-Agencia de Ciencia y Tecnología de la Región de Murcia), by the national research project MTM2015- 64382-P (MINECO/FEDER) and by NSF grant DMS-1719410. 


\section{REFERENCES}

[1] A.A. Abushama and B. Bialecki, Modified nodal cubic spline collocation for Poisson's equation. SIAM J. Numer. Anal. 46 (2008) 397-418.

[2] S. Amat and J. Liandrat, On the stability of the PPH nonlinear multiresolution. Appl. Comput. Harmon. Anal. 18 (2005) 198-206.

[3] S. Amat and J. Ruiz, New WENO smoothness indicators computationally efficient in the presence of corner discontinuities. J. Sci. Comput. 71 (2017) 1265-1302.

[4] S. Amat, F. Aràndiga, A. Cohen, R. Donat, G. Garcia and M. von Oehsen, Data compression with ENO schemes: a case study. Appl. Comput. Harmon. Anal. 11 (2001) 273-288.

[5] S. Amat, S. Busquier and J.C. Trillo, On multiresolution schemes using a stencil selection procedure: applications to ENO schemes. Numer. Algorithms 44 (2007) 45-68.

[6] S. Amat, K. Dadourian, J. Liandrat, J. Ruiz and J.C. Trillo, On a class of L1-stable nonlinear cell-average multiresolution schemes. J. Comput. Appl. Math. 234 (2010) 1129-1139.

[7] S. Amat, S. Busquier, M. Legaz, F. Manzano and J. Ruiz, Reciprocal polynomial extrapolation vs Richardson extrapolation for singular perturbed boundary problems. Numer. Algorithms 61 (2012) 631-647.

[8] S. Amat, J. Liandrat, J. Ruiz and J. Trillo, On a compact non-extrapolating scheme for adaptive image interpolation. J. Franklin Inst. 349 (2012) 1637-1647.

[9] S. Amat, J. Ruiz and J.C. Trillo, Adaptive interpolation of images using a new nonlinear cell-average scheme. Math. Comput. Simul. 82 (2012) 1586-1596.

[10] S. Amat, K. Dadourian, J. Liandrat and J. Trillo, High order nonlinear interpolatory reconstruction operators and associated multiresolution schemes. J. Comput. Appl. Math. 253 (2013) 163-180.

[11] S. Amat, J. Ruiz and J.C. Trillo, Improving the compression rate versus $L^{1}$ error ratio in cell-average error control algorithms. Numer. Algorithms 67 (2014) 145-162.

[12] S. Amat, J. Liandrat, J. Ruiz and J. Trillo, On a family of nonlinear cell-average multiresolution schemes for image processing. Math. Comput. Simul. 118 (2015) 30-48.

[13] S. Amat, J. Liandrat, J. Ruiz and J.C. Trillo, On a power WENO scheme with improved accuracy near discontinuities. SIAM J. Sci. Comput. 39 (2017) A2472-A2507.

[14] S. Amat, J. Ruiz and J.C. Trillo, On an algorithm to adapt spline approximations to the presence of discontinuities. Numer. Algorithms 80 (2019) 903-936.

[15] F. Aràndiga and R. Donat, Nonlinear multiscale decompositions: the approach of A. Harten. Numer. Algorithms 23 (2000) 175-216.

[16] F. Arandiga, A. Cohen, R. Donat, N. Dyn and B. Matei, Approximation of piecewise smooth functions and images by edgeadapted (ENO-EA) nonlinear multiresolution techniques. Special Issue on Mathematical Imaging - Part II. Appl. Comput. Harmon. Anal. 24 (2008) 225-250.

[17] F. Aràndiga, A. Belda and P. Mulet, Point-value WENO multiresolution applications to stable image compression. J. Sci. Comput. 43 (2010) 158-182.

[18] A.K.B. Chand and G.P. Kapoor, Generalized cubic spline fractal interpolation functions. SIAM J. Numer. Anal. 44 (2006) $655-676$.

[19] A. Cohen, N. Dyn and B. Matei, Quasi linear subdivision schemes with applications to ENO interpolation. Appl. Comput. Harmon. Anal. 15 (2003) 89-116.

[20] O. Davydov and L.L. Schumaker, Stable approximation and interpolation with $C^{1}$ quartic bivariate splines. SIAM J. Numer. Anal 39 (2002) 1732-1748.

[21] C. de Boor, A Practical Guide to Splines. SIAM, Springer-Verlag New York 27 (1980).

[22] R. Devore, Nonlinear approximation. Acta Numer. 7 (1998) 51-150.

[23] M.S. Floater and M.-J. Lai, Polygonal spline spaces and the numerical solution of the Poisson equation. SIAM J. Numer. Anal. 54 (2016) 797-824.

[24] J. Foster and F.B. Richards, Gibbs-Wilbraham splines. Constr. Approx. 11 (1995) 37-52.

[25] D. Gottlieb and C.-W. Shu, On the Gibbs phenomenon and its resolution. SIAM Rev. 39 (1997) $644-668$.

[26] A. Harten, Multiresolution representation of data: a general framework. SIAM J. Numer. Anal. 33 (1996) $1205-1256$.

[27] A. Harten, Multiresolution representation of data II. SIAM J. Numer. Anal. 33 (1996) 1205-1256.

[28] A. Harten and S. Osher, Uniformly high-order accurate nonoscillatory schemes. I. SIAM J. Numer. Anal. 24 (1987) $279-309$.

[29] A. Harten, B. Engquist, S. Osher and S.R. Chakravarthy, Uniformly high order accurate essentially non-oscillatory schemes, III. J. Comput. Phys. 71 (1987) 231-303.

[30] F. Richards, A Gibbs phenomenon for spline functions. J. Approx. Theory 66 (1991) 334-351.

[31] L.L. Schumaker, Spline Functions: Computational Methods. SIAM, Springer-Verlag New York (2015).

[32] D. Schweikert, An interpolation curve using splines in tension. J. Math. Phys. 45 (1966) 312-317.

[33] S. Serna and A. Marquina, Power ENO methods: a fifth-order accurate weighted power ENO method. J. Comput. Phys. 194 (2004) 632-658.

[34] C.-W. Shu, Order ENO and WENO Schemes for Computational Fluid Dynamics. Springer, Berlin, Heidelberg (1999) 439-582.

[35] C.-W. Shu and S. Osher, Efficient implementation of essentially non-oscillatory shock-capturing schemes. J. Comput. Phys. 77 (1988) 439-471. 
[36] C.-W. Shu and S. Osher, Efficient implementation of essentially non-oscillatory shock-capturing schemes II. J. Comput. Phys. 83 (1989) 32-78.

[37] H. Späth, Exponential spline interpolation. Computing 4 (1969) 225-233.

[38] R.A. Usmani, Inversion of Jacobi's tridiagonal matrix. Comput. Math. App. 27 (1994) 59-66.

[39] Z. Zhang and C.F. Martin, Convergence and Gibbs' phenomenon in cubic spline interpolation of discontinuous functions. J. Comput. Appl. Math. 87 (1997) 359-371. 